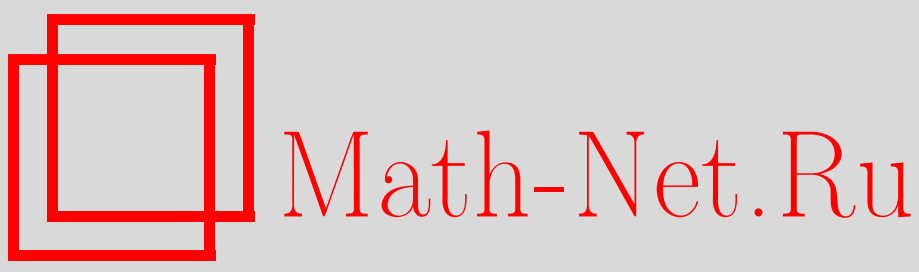

В. Ф. Кириченко, В. В. Коннов, Почти келеровы многообразия гиперболического типа, Изв. РАН. Сер. матем., 2003, том 67, выпуск 4, 21-66

DOI: https://doi.org/10.4213/im442

Использование Общероссийского математического портала Math-Net.Ru подразумевает, что вы прочитали и согласны с пользовательским соглашением

http://www . mathnet.ru/rus/agreement

Параметры загрузки:

IP: 44.207 .124 .84

26 апреля 2023 г., 15:09:04 
УДК 513.7

\author{
В.Ф. Кириченко, В.В. Коннов
}

\title{
Почти келеровы многообразия гиперболического типа
}

\begin{abstract}
Изучена геометрия вполне вещественных подмногообразий почти келеровых многообразий гиперболического типа. Основное внимание уделено изучению геометрии многообразия невырожденных нуль-пар вещественного проективного пространства. Получена полная классификация вполне геодезических лагранжевых подмногообразий этого многообразия и приведено их конструктивное построение.

Библиограффия: 14 наименований.
\end{abstract}

Почти эрмитовы структуры гиперболического типа ( $A \mathscr{H}_{1}$-структуры) являются очень интересным и в то же время сравнительно малоизученным объектом исследования обобщенной эрмитовой геометрии. Это объясняется тем, что до последнего времени было известно весьма мало примеров таких структур, а внимание исследователей они привлекали, главным образом, благодаря их поверхностному сходству с классическими почти эрмитовыми структурами. Ситуация сушественно изменилась после того, как первому из авторов удалось доказать, что задание почти эрмитовой структуры гиперболического типа на римановом многообразии равносильно заданию на нем почти антикватернионной структуры [1], [2]. Этот результат позволил ввести в рассмотрение обширные классы конкретных примеров $A \mathscr{H}_{1}$-структур. Важным примером таких структур являются $A \mathscr{H}_{1}$-структуры, канонически возникающие на пространстве касательного расслоения к риманову многообразию [2]. Такие структуры обладают тем важным свойством, что их фундаментальная форма всегда замкнута, а следовательно, определяет симплектическую структуру. $A \mathscr{H}_{1}$-структуры с замкнутой фундаментальной формой, по аналогии с классическим случаем, называются почти келеровым.ми.

Другим важным примером многообразий, несуших $A \mathscr{H}_{1}$-структуру, является пространство $\mathbb{R} P_{n} \diamond \mathbb{R} P_{n}$ (невырожденных) нуль-пар вещественного проективного пространства. Первым автором получен весьма общий результат, из которого следует, что келерово (и даже почти келерово) многообразие гиперболического типа, удовлетворяющее аксиоме голоморфных плоскостей, локально эквивалентно пространству невырожденных нуль-пар $\mathbb{R} P_{n} \diamond \mathbb{R} P_{n}$, рассматриваемому как однородное пространство $\mathrm{GL}(n+1, \mathbb{R}) / \mathrm{GL}(n, \mathbb{R}) \times \mathrm{GL}(1, \mathbb{R})$. Таким образом, это пространство является гиперболическим аналогом комплексных пространственных форм в эрмитовой геометрии [3]. Однако до настоящего времени каноническая $A \mathscr{H}_{1}$-структура пространства нуль-пар конструктивно практически не изучалась.

(C) В.Ф. КиРиченко, В.В. Коннов, 2003 
В частности, ничего не было известно о структуре вполне вещественных подмногообразий пространства нуль-пар. В то же время такие подмногообразия, будучи лагранжевыми подмногообразиями соответствуюшей симплектической структуры, представляют существенный интерес.

В настояшей работе изучается геометрия весьма широкого класса $A \mathscr{H}_{1}$-многообразий, а именно квазикелеровых $A \mathscr{H}_{1}$-многообразий, включающего все упомянутые выше классы $A \mathscr{H}_{1}$-многообразий. Особое внимание уделяется изучению вполне вещественных подмногообразий квазикелеровых $A \mathscr{H}_{1}$-многообразий. Доказано, что через каждую точку такого многообразия $M^{2 n}$ в любом $n$-мерном вполне вещественном направлении проходит (вообще говоря, не единственное) вполне вешественное подмногообразие тогда и только тогда, когда $M^{2 n}$ - почти келерово $A \mathscr{H}_{1}$-многообразие. Этот результат проясняет геометрический смысл замкнутости фундаментальной формы квазикелерова $A \mathscr{H}_{1}$-многообразия. Доказано также, что если $\operatorname{dim} M>4$, то в классе вполне вещественных подмногообразий, проходящих через данную точку в данном вполне вешественном направлении, присутствует и притом единственное вполне геодезическое подмногообразие тогда и только тогда, когда $M$ локально эквивалентно пространству $\mathbb{R} P_{n} \diamond \mathbb{R} P_{n}$ либо двойному евклидову пространству $\mathbb{R}^{n} \otimes \mathbb{R}^{n}$. Более того, оказывается, что это вполне вешественное подмногообразие является пространством постоянной кривизны. Рассмотрены контактные аналоги упомянутых результатов, а также приложения этих результатов к геометрии главных $T^{1}$-расслоений.

$\mathrm{B} \S 2$ изучается геометрия канонической $A \mathscr{H}_{1}$-структуры пространства $\mathbb{R} P_{n} \diamond$ $\mathbb{R} P_{n}$ невырожденных нуль-пар. Доказано, что относительно этой структуры многообразие $\mathbb{R} P_{n} \diamond \mathbb{R} P_{n}$ является $\mathscr{K}_{1}$-многообразием ненулевой постоянной голоморфной секционной кривизны (т. е. натуральной пространственной формой), причем этими свойствами указанная структура определена с точностью до локальной эквивалентности. Получена полная классификация вполне геодезических вполне вещественных подмногообразий этого пространства и, более того, получено исчерпьвающее описание их строения, а также приводится их конструктивное построение. В частности, дается исчерпьвающая геометрическая характеризация вполне изотропных подмногообразий максимальной размерности, которые с необходимостью являются вполне геодезическими и лагранжевыми. Получены примеры компактных лагранжевых подмногообразий многообразия нуль-пар (заметим, что само многообразие $\mathbb{R} P_{n} \diamond \mathbb{R} P_{n}$ некомпактно).

\section{§1. Общая теория почти эрмитовых многообразий гиперболического типа}

1.1. Структурные уравнения $A \mathscr{H}_{1}$-многообразий. Пусть $M$-гладкое многообразие, $\mathfrak{X}(M)-C^{\infty}(M)$-модуль гладких векторных полей на $M$ и $d$ - оператор внешнего дифференцирования. Все многообразия, тензорные поля и подобные объекты предполагаются гладкими класса $C^{\infty}$. Напомним [6], что почти әрмитовой структурой гиперболического типа (А $\mathscr{H}_{1}$-структурой), или, в иной 
терминологии, почти параэрмитовой структурой [7] на многообразии $M$ называется пара $\{g, I\}$, где $g=\langle\cdot, \cdot\rangle$ - псевдориманова метрика на $M$ и $I$ - структура почти произведения, т. е. инволютивный автоморфизм касательного пучка многообразия $M$. При этом должно выполняться тождество

$$
\langle I X, I Y\rangle=-\langle X, Y\rangle, \quad X, Y \in \mathfrak{X}(M) .
$$

Из этих условий следует, что тензор $\Omega(X, Y)=\langle X, I Y\rangle$ кососимметричен; он называется фундаментальной формой структуры. Как и в случае симплектической структуры, необходимыми условиями существования $A \mathscr{H}_{1}$-структуры на многообразии является его четномерность и ориентируемость. Пусть $\nabla$ - риманова связность метрики $g$. Почти эрмитова структура $\{g, I\}$ гиперболического типа называется:

- квазикелеровой (Q $\mathscr{K}_{1}$-структурой), если $\nabla_{X}(I) Y-\nabla_{I X}(I)(I Y)=0$;

- почти келеровой (А К 1 -структурой), если $d \Omega=0$;

- келеровой ( $\mathscr{K}_{1}$-структурой), если $\nabla I=0$.

Легко видеть, что эти определения согласуются с определениями обобщенных квазикелеровых, обобщенных почти келеровых и обобщенных келеровых структур, введенных в работах первого автора (см., например, [4], [5]), и в то же время являются естественными аналогами соответствующих определений в классической эрмитовой геометрии [8]. Как и в классическом случае, легко доказывается, что $\mathscr{K}_{1} \subset A \mathscr{K}_{1} \subset Q \mathscr{K}_{1}$. Все рассматриваемые ниже почти эрмитовы структуры подразумеваются структурами гиперболического типа.

Пусть $M-2 n$-мерное $A \mathscr{H}_{1}$-многообразие, т. е. многообразие, снабженное $A \mathscr{H}_{1}$ структурой; $n$-мерное подмногообразие $N \subset M$ называется вполне вещественныл.м, если $\left.\Omega\right|_{N}=0$, т.е. $\langle X, I Y\rangle=0, X, Y \in \mathfrak{X}(N)$.

ОПРЕДЕЛЕНИЕ 1 . Говорят, что $A \mathscr{H}_{1}$-многообразие $M$ удовлетворяет аксиоме вполне вещественных подмногообразий, если в каждой точке $p \in M$ для каждого вполне вещественного подпространства $L \subset T_{p}(M)$ существует вполне вешественное подмногообразие $N \subset M$, проходящее через точку $p$ и такое, что $L=T_{p}(N)$. Если каждое такое подмногообразие может быть выбрано вполне геодезическим, то говорят, что многообразие $M$ удовлетворяет аксиоме вполне вещественных плоскостей.

ЗАмечАниЕ 1 . Очевидно, вполне вешественные подмногообразия $A \mathscr{K}_{1}$-многообразия есть не что иное, как подмногообразия Лагранжа относительно симплектической структуры $\Omega$.

Пусть $\mathscr{S}=\{g=\langle\cdot, \cdot\rangle, I\}-A \mathscr{H}_{1}$-структура на многообразии $M^{2 n}, p \in M$. Тогда в касательном пространстве $T_{p}(M)$ можно построить ортонормированньй репер $\left(p, e_{1}, \ldots, e_{n}, I e_{1}, \ldots, I e_{n}\right)$, который будем называть вещественно адаптированным репером. С другой стороны, в модуле $\mathfrak{X}(M) \otimes \mathrm{D}$, где $\mathrm{D}$ - кольцо двойных чисел, внутренним образом определены два взаимно дополнительных проектора $\sigma=\frac{1}{2}(\mathrm{id}+i I)$ и $\bar{\sigma}=\frac{1}{2}(\mathrm{id}-i I)$ на собственные подмодули эндоморфизма $I$, 
отвечающие собственным значениям $i$ и $-i$ соответственно, где $i$ - мнимая единица кольца D. Следовательно, в пространстве $T_{p}(M) \otimes \mathrm{D}$ можно построить репер $\left(p, \varepsilon_{1}, \ldots, \varepsilon_{n}, \varepsilon_{\hat{1}}, \ldots, \varepsilon_{\hat{n}}\right)$, где $\varepsilon_{a}=\sqrt{2} \sigma\left(e_{a}\right), \varepsilon_{\hat{a}}=\sqrt{2} \bar{\sigma}\left(e_{a}\right)$, состоящий из собственных векторов оператора $I_{p}$. Такой репер называется $A$-репером. Легко видеть, что матрицы компонент тензоров $I_{p}$ и $g_{p}$ в $A$-репере имеют соответственно вид

$$
\left(I_{j}^{i}\right)=\left(\begin{array}{cc}
i I_{n} & 0 \\
0 & -i I_{n}
\end{array}\right), \quad\left(g_{i j}\right)=\left(\begin{array}{cc}
0 & I_{n} \\
I_{n} & 0
\end{array}\right),
$$

где $I_{n}$ - единичная матрища порядка $n$. Хорошо известно [4], [5], что совокупность таких реперов определяет $G$-структуру на $M$ со структурной группой $U(n, \mathrm{D})$, представленной матрицами вида $\left(\begin{array}{cc}A & 0 \\ 0 & \bar{A}\end{array}\right)$, где $A \in U(n, \mathrm{D})$. Эта $G$-структура называется присоединенной. На протяжении всей работы будем подразумевать, что индексы $i, j, k, \ldots$ пробегают значения от 1 до $2 n$, индексы $a, b, c, d, f, g, \ldots-$ значения от 1 до $n$, и положим $\hat{a}=a+n, \widehat{\hat{a}}=a$. Поскольку $I$ и $g$ - тензоры типов $(1,1)$ и $(2,0)$ соответственно, их компоненты на пространстве расслоения всех комплексных реперов над $M$ удовлетворяют уравнениям

$$
\begin{gathered}
d I_{j}^{i}+I_{k}^{i} \omega_{j}^{k}-I_{j}^{k} \omega_{k}^{i}=I_{j, k}^{i} \omega^{k} \\
d g_{i j}+g_{k j} \omega_{i}^{k}+g_{i k} \omega_{j}^{k}=g_{i j, k} \omega^{k}
\end{gathered}
$$

где $\left\{\omega^{i}\right\},\left\{\omega_{j}^{i}\right\}$ - компоненты форм смещения и римановой связности $\nabla$ соответственно, $I_{j, k}^{i}, g_{i j, k}$ - компоненты ковариантного дифференциала тензоров $I$ и $g$ в этой связности соответственно. Более того, в силу определения римановой связности $\nabla g=0$, а значит,

$$
g_{i j, k}=0 \text {. }
$$

С учетом равенств (1) и (3) соотношения (2) на пространстве присоединенной $G$-структуры перепишутся в форме

$$
\begin{gathered}
I_{b, k}^{a}=0, \quad I_{\hat{b}, k}^{\hat{a}}=0, \\
\omega_{\hat{b}}^{a}=\frac{1}{2} i I_{\hat{b}, k}^{a} \omega^{k}, \quad \omega_{b}^{\hat{a}}=-\frac{1}{2} i I_{b, k}^{\hat{a}} \omega^{k}, \\
\omega_{j}^{i}+\omega_{\hat{i}}^{\hat{j}}=0 .
\end{gathered}
$$

Кроме того, заметим, что в силу вещественности соответствующих форм и тензоров $\bar{\omega}^{i}=\omega^{\hat{i}}, \bar{\omega}_{j}^{i}=\omega_{\hat{j}}^{\hat{i}}, \overline{\nabla I}_{j, k}^{i}=\nabla I_{\hat{j}, \hat{k}}^{\hat{i}}$, где $t \rightarrow \bar{t}$-оператор естественного сопряжения. Несложно подсчитать, что $A \mathscr{H}_{1}$-структура является квазикелеровой тогда и только тогда, когда на пространстве присоединенной $G$-структуры

$$
I_{\hat{b}, c}^{a}=0 .
$$

При этом она является почти келеровой тогда и только тогда, когда

$$
I_{\hat{b}, c}^{a}=0, \quad I^{[a b, c]}=0,
$$


и является келеровой тогда и только тогда, когда

$$
I_{\hat{b}, c}^{a}=0, \quad I_{\hat{b}, \hat{c}}^{a}=0 .
$$

Здесь и в дальнейшем по индексам, заключенньм в квадратные (соответственно, круглые) скобки подразумевается альтернирование (соответственно, симметризация).

С учетом (4) нетрудно выяснить, что первая группа структурных уравнений римановой связности

$$
d \omega^{i}=\omega_{j}^{i} \wedge \omega^{j}
$$

на пространстве присоединенной $G$-структуры $A \mathscr{H}_{1}$-многообразия запишется в следующей форме, называемой первой группой структурных уравнений А期многообразия:

$$
\begin{aligned}
& d \omega^{a}=\omega_{b}^{a} \wedge \omega^{b}+B_{c}^{a b} \omega^{c} \wedge \omega_{b}+B^{a b c} \omega_{b} \wedge \omega_{c}, \\
& d \omega_{a}=-\omega_{a}^{b} \wedge \omega_{b}+B_{a b}^{c} \omega_{c} \wedge \omega^{b}+B_{a b c} \omega^{b} \wedge \omega^{c},
\end{aligned}
$$

где $\omega_{i}=g_{i j} \omega^{j}, B_{c}^{a b}=\frac{1}{2} i I_{\hat{b}, c}^{a}, B^{a b c}=-\frac{1}{2} i I_{[\hat{b}, \hat{c}]}^{a}, B_{a b}^{c}=-\frac{1}{2} i I_{b, \hat{c}}^{\hat{a}}, B_{a b c}=\frac{1}{2} i I_{[b, c]}^{\hat{a}}$. При этом

$$
\begin{gathered}
\overline{\omega_{b}^{a}}=-\omega_{a}^{b}, \quad \overline{B_{c}^{a b}}=B_{a b}^{c}, \quad \overline{B^{a b c}}=B_{a b c}, \\
B_{c}^{(a b)}=0, \quad B^{a(b c)}=0 .
\end{gathered}
$$

Более того, с учетом (4) нетрудно получить, что

$$
I_{\hat{b}, \hat{c}}^{a}=4 i \widetilde{B}^{c a b},
$$

где $\widetilde{B}^{c a b}=\frac{1}{2}\left(B^{c a b}-B^{a b c}-B^{b c a}\right)$. Согласно (5)-(7) справедливо

ПРЕДЛОЖЕНИЕ 1. АН̈ ко тогда, когда $B_{c}^{a b}=0$, является почти келеровой тогда и только тогда, когда $B_{c}^{a b}=0, B^{[a b c]}=0$, иявляется келеровой тогда и только тогда, когда $B^{a b c}=0, \quad B_{c}^{a b}=0$.

ЗАмечАниЕ 2 . Очевидно, почти келеровость $Q \mathscr{K}_{1}$-многообразия равносильна соотношению $B^{a b c}=\widetilde{B}^{a b c}$.

Пусть, в частности, $M-Q \mathscr{K}_{1}$-многообразие. Стандартная процедура дифференциального продолжения соотношений (9) с учетом предложения 1 приводит ко второй группе структурных уравнений Q $\mathscr{K}_{1}$-структуры:

$$
\begin{aligned}
d \omega_{b}^{a}= & \omega_{c}^{a} \wedge \omega_{b}^{c}+\left(2 B^{a d h} B_{h b c}+A_{b c}^{a d}\right) \omega^{c} \wedge \omega_{d} \\
& +B_{b c d}^{a} \omega^{c} \wedge \omega^{d}-B_{b}^{a c d} \omega_{c} \wedge \omega_{d},
\end{aligned}
$$


где $\left\{A_{b c}^{a d}\right\}$ - семейство функций на пространстве присоединенной $G$-структуры, служащих компонентами так называемого тензора кривизны присоединенной $Q$-алгебры [5], или структурного тензора второго рода, причем

$$
A_{[b c]}^{a d}=0, \quad A_{b c}^{[a d]}=2 B_{(b c) h} B^{h a d} .
$$

Кроме того,

$$
\begin{aligned}
& d B^{a b c}-B^{h b c} \omega_{h}^{a}-B^{a h c} \omega_{h}^{b}-B^{a b h} \omega_{h}^{c}=B_{h}^{a b c} \omega^{h}+B^{a b c h} \omega_{h}, \\
& d B_{a b c}+B_{h b c} \omega_{a}^{h}+B_{a h c} \omega_{b}^{h}+B_{a b h} \omega_{c}^{h}=B_{a b c h} \omega^{h}+B_{a b c}^{h} \omega_{h},
\end{aligned}
$$

где $B_{d}^{a b c}, B^{a b c d}, B_{a b c d}, B_{a b c}^{d}$ - подходящие функции на пространстве присоединенной $G$-структуры. Отсюда, в частности, следует, что наборы функций $\left\{B^{a b c}\right\}$ и $\left\{B_{a b c}\right\}$ являются наборами компонент взаимно сопряженных тензоров на $M$, которые мы назовем структурными тензорами первого рода.

Сравнивая соотношения (12) со второй группой структурных уравнений римановой связности

$$
d \omega_{j}^{i}=\omega_{k}^{i} \wedge \omega_{j}^{k}+\frac{1}{2} R_{j k l}^{i} \omega^{k} \wedge \omega^{l},
$$

где $R_{j k l}^{i}$ - компоненты тензора Римана-Кристоффеля, в силу линейной независимости базисных форм получим, что на пространстве присоединенной $G$-структуры

$$
R_{\hat{a} b c d}=2 B_{b c d}^{a}, \quad R_{\hat{a} b c \hat{d}}=2 B^{a d h} B_{h b c}-4 \widetilde{B}^{d a h} \widetilde{B}_{c b h}+A_{b c}^{a d} .
$$

Кроме того, с учетом (5), (6) и (11) имеем

$$
\omega^{a b} \equiv \omega_{\hat{b}}^{a}=2 \widetilde{B}^{h a b} \omega_{h}, \quad \omega_{a b} \equiv \omega_{b}^{\hat{a}}=2 \widetilde{B}_{h a b} \omega^{h} .
$$

Дифференциальное продолжение этих соотношений с учетом (9), (14) и (15) приводит к равенствам

$$
R_{a b c d}=4 \widetilde{B}_{[d|a b| c]}, \quad R_{\hat{a} \hat{b} c d}=4 \widetilde{B}^{h a b} B_{h c d},
$$

а также к соотношениям, полученным из (16) и (18) с учетом классических свойств симметрии и вещественности тензора Римана-Кристоффеля.

ОПРЕДЕЛЕНИЕ 2. А $\mathscr{H}_{1}$-многообразие называется многообразием точечно постоянной голоморфной секционной кривизны с, если

$$
\forall X \in \mathscr{L} \Longrightarrow\langle R(X, I X) X, I X\rangle=c\|X\|^{4} .
$$

$\mathscr{K}_{1}$-многообразие точечно постоянной голоморфной секционной кривизны называется натуральной пространственной формой [5].

Пусть $M-A \mathscr{H}_{1}$-многообразие точечно постоянной голоморфной секционной кривизны $c$. Соотношение (19) на пространстве присоединенной $G$-структуры запишется в форме

$$
4 R_{\hat{a} b f \hat{d}} X^{\hat{a}} X^{b} X^{f} X^{\hat{d}}=-4 c g_{\hat{a} b} g_{f \hat{d}} X^{\hat{a}} X^{b} X^{f} X^{\hat{d}} .
$$

С учетом второго равенства в (1) это соотношение можно переписать в виде

$$
\left(R_{\hat{a} b f \hat{d}}+c \delta_{b}^{a} \delta_{f}^{d}\right) X^{\hat{a}} X^{b} X^{f} X^{\hat{d}}=0 .
$$

Поляризация этого соотношения приводит нас к следующему результату. 
ПРЕДЛОЖЕНИЕ 2. АЯН 1 -многообразие является многообразием точечно постоянной голоморфной секционной кривизны с тогда и только тогда, когда

$$
R_{(b f)}^{(a)}=-\frac{c}{2} \tilde{\delta}_{b f}^{a d},
$$

əде $\tilde{\delta}_{b f}^{a d}=\delta_{b}^{a} \delta_{f}^{d}+\delta_{f}^{a} \delta_{b}^{d}$.

Пусть, в частности, $M-Q \mathscr{K}_{1}$-многообразие. Тогда с учетом второго равенства из (16) соотношение $(20)$ принимает вид $A_{b f}^{(a d)}=4 \widetilde{B}^{(a d) h} \widetilde{B}_{(b f) h}-\frac{c}{2} \tilde{\delta}_{b f}^{a d}$. Используя это соотношение и второе равенство из (13), после упрощений получим

ПРЕДЛОЖЕНИЕ 3. Q К्К -многообразие является многообразием точечно постоянной голоморфной секционной кривизны с тогда и только тогда, когда

$$
A_{b f}^{a d}=4 \widetilde{B}^{d a h} B_{(b f) h}-\frac{c}{2} \tilde{\delta}_{b f}^{a d} .
$$

1.2. Вполне вещественные подмногообразия $Q \mathscr{K}_{1}$-многообразий. Пусть $M^{2 n}-Q \mathscr{K}_{1}$-многообразие и $N^{n} \subset M^{2 n}$-его вполне вещественное подмногообразие. Фиксируем точку $p \in N$. Допустим, что $\left(p, e_{1}, \ldots, e_{n}\right)$-ортонормированньй репер касательного пространства $T_{p}(N)$, который однозначно достраивается до вешественно-адаптированного репера $\left(p, e_{1}, \ldots, e_{n}, I e_{1}, \ldots, I e_{n}\right)$ касательного пространства $T_{p}(M)$. Рассмотрим дуальньй корепер $\left(p, \theta^{1}, \ldots, \theta^{n}, \theta^{\hat{1}}, \ldots, \theta^{\hat{n}}\right)$. Очевидно, сужение на $N$ семейства всех вешественно-адаптированных реперов многообразия $M$ определяет $G$-структуру со структурной группой $U(n, \mathrm{D})$, в котором семейство $\mathscr{B}$ вешественно-адаптированных реперов указанного выше вида образует подструктуру со структурной группой $O(n, \mathbb{R})$, пространство которой задается системой Пфаффа

$$
\theta^{\hat{a}}=0 .
$$

Нам, однако, удобнее будет иметь дело с главным расслоением $A$-реперов $\left(p, \varepsilon_{1}, \ldots\right.$ $\left.\ldots, \varepsilon_{n}, \varepsilon_{\hat{1}}, \ldots, \varepsilon_{\hat{n}}\right)$, порожденных реперами семейства $\mathscr{B}$. Напомним, что $\varepsilon_{a}=$ $\frac{1}{\sqrt{2}}\left(e_{a}+i I e_{a}\right), \quad \varepsilon_{\hat{a}}=\frac{1}{\sqrt{2}}\left(e_{a}-i I e_{a}\right)$. Поэтому матрица $C=\left(C_{j}^{i}\right)$ перехода от вещественно-адаптированного репера к соответствующему $A$-реперу имеет вид

$$
\left(C_{j}^{i}\right)=\frac{1}{\sqrt{2}}\left(\begin{array}{cc}
I_{n} & I_{n} \\
i I_{n} & -i I_{n}
\end{array}\right) .
$$

Следовательно, если $\left(p, \omega^{1}, \ldots, \omega^{n}, \omega^{\hat{1}}, \ldots, \omega^{\hat{n}}\right)$ - корепер, дуальный $A$-реперу, то $\omega^{i}=D_{j}^{i} \theta^{j}$, где $D=\left(D_{j}^{i}\right)$ - матрица, обратная матрице $C$. Очевидно,

$$
\left(D_{j}^{i}\right)=\frac{1}{\sqrt{2}}\left(\begin{array}{cc}
I_{n} & i I_{n} \\
I_{n} & -i I_{n}
\end{array}\right) .
$$

Поэтому

$$
\omega^{a}=\frac{1}{\sqrt{2}}\left(\theta^{a}+i \theta^{\hat{a}}\right), \quad \omega^{\hat{a}}=\frac{1}{\sqrt{2}}\left(\theta^{a}-i \theta^{\hat{a}}\right) .
$$


В частности, система Пфаффа (22) может быть переписана в форме

$$
\omega^{a}=\omega_{a}
$$

Далее, первая группа структурных уравнений римановой связности на пространстве расслоения вешественно-адаптированных реперов многообразия $M$ имеет вид

$$
d \theta^{i}=\theta_{j}^{i} \wedge \theta^{j},
$$

где $\left\{\theta_{j}^{i}\right\}$ - компоненты формы римановой связности на этом пространстве. Поскольку $\theta^{i}=C_{j}^{i} \omega^{j}$, это соотношение можно также переписать в форме $C_{j}^{i} d \omega^{j}=$ $\theta_{j}^{i} \wedge C_{k}^{j} \omega^{k}$, или

$$
d \omega^{i}=\left(D_{m}^{i} \theta_{k}^{m} C_{j}^{k}\right) \wedge \omega^{j} .
$$

С другой стороны, в силу (8) имеем $d \omega^{i}=\omega_{j}^{i} \wedge \omega^{j}$. Сравнивая (28) с этим соотношением, получим, что $\left(D_{m}^{i} \theta_{k}^{m} C_{j}^{k}-\omega_{j}^{i}\right) \wedge \omega^{j}=0$, и в силу леммы Картана

$$
\omega_{j}^{i}=D_{m}^{i} \theta_{k}^{m} C_{j}^{k}+C_{j k}^{i} \omega^{k},
$$

где $\left\{C_{j k}^{i}\right\}$ - система функций на пространстве расслоения $A$-реперов, симметричная по нижним индексам.

Лемма 1. В принятых обозначениях $C_{j k}^{i}=0$.

ДокАЗАТЕЛЬСтво. С учетом параллельности метрического тензора в римановой связности имеем

$$
d \tilde{g}_{i j}+\tilde{g}_{k j} \theta_{i}^{k}+\tilde{g}_{i k} \theta_{j}^{k}=0, \quad d g_{i j}+g_{k j} \omega_{i}^{k}+g_{i k} \omega_{j}^{k}=0,
$$

где $\left\{\tilde{g}_{i j}\right\}$ и $\left\{g_{i j}\right\}$ - компоненты метрического тензора $g$ многообразия $M$ в вешественно-адаптированном репере и $A$-репере соответственно. Поскольку $g$ - тензор типа $(2,0)$, то $g_{i j}=C_{i}^{k} C_{j}^{m} \tilde{g}_{k m}$. В силу этого второе соотношение в $(30)$ примет вид

$$
\tilde{g}_{m s} C_{k}^{m} C_{j}^{s} \omega_{i}^{k}+\tilde{g}_{m s} C_{i}^{m} C_{k}^{s} \omega_{j}^{k}=0 .
$$

Подставляя сюда (29), после упрощений получим

$$
\tilde{g}_{m s} C_{j}^{s} \theta_{t}^{m} C_{i}^{t}+\tilde{g}_{m s} C_{i}^{m} \theta_{t}^{s} C_{j}^{t}+\left(\tilde{g}_{k j} C_{i t}^{k}+\tilde{g}_{i k} C_{j t}^{k}\right) \omega^{t}=0
$$

Но в силу первого соотношения в (30) сумма первых двух слагаемых равна нулю, и в силу линейной независимости базисных форм имеем $\tilde{g}_{k j} C_{i t}^{k}+\tilde{g}_{i k} C_{j t}^{k}=0$. Дважды делая в этом соотношении циклическую перестановку индексов $i, j$ и $t$, почленно сложим первые два соотношения и почленно вычтем третье: $\tilde{g}_{k j} C_{i t}^{k}=0$. В силу невырожденности матрицы метрического тензора отсюда следует, что $C_{i t}^{k}=0$.

С учетом леммы 1 соотношение (29) можно переписать в форме

$$
\theta_{j}^{i}=C_{m}^{i} \omega_{k}^{m} D_{j}^{k}
$$


Выясним условия интегрируемости системы (22). В силу (27) условия $\left.d \theta^{\hat{a}}\right|_{N}=0$ принимают вид $\theta_{b}^{\hat{a}} \wedge \theta^{b}=0$, откуда по лемме Картана на подмногообразии $N$ должно выполняться соотношение

$$
\theta_{b}^{\hat{a}}=H_{b c}^{\hat{a}} \theta^{c}
$$

где $H_{b c}^{\hat{a}}=H_{c b}^{\hat{a}}-$ компоненты второй квадратичной формы погружения $N \subset M$. Заметим, что в силу (31)

$$
\theta_{b}^{\hat{a}}=C_{h}^{\hat{a}} \omega_{g}^{h} D_{b}^{g}+C_{\hat{h}}^{\hat{a}} \omega_{g}^{\hat{h}} D_{b}^{g}+C_{h}^{\hat{a}} \omega_{\hat{g}}^{h} D_{b}^{\hat{g}}+C_{\hat{h}}^{\hat{a}} \omega_{\hat{g}}^{\hat{h}} D_{b}^{\hat{g}}
$$

откуда с учетом (23) и (24) получаем

$$
\theta_{b}^{\hat{a}}=\frac{i}{2}\left(\omega_{b}^{a}-\omega_{b}^{\hat{a}}+\omega_{\hat{b}}^{a}-\omega_{\hat{b}}^{\hat{a}}\right)
$$

В частности, соотношение (32) можно переписать в форме

$$
\omega_{b}^{a}-\omega_{b}^{\hat{a}}+\omega_{\hat{b}}^{a}-\omega_{\hat{b}}^{\hat{a}}=2 i H_{b c}^{\hat{a}} \theta^{c} .
$$

Аналогично,

$$
\theta_{b}^{a}=\frac{1}{2}\left(\omega_{b}^{a}+\omega_{b}^{\hat{a}}+\omega_{\hat{b}}^{a}+\omega_{\hat{b}}^{\hat{a}}\right) .
$$

Заметим, что соотношение (33) можно записать в форме

$$
\left(\omega_{b}^{a}+\omega_{a}^{b}\right)+\left(\omega^{a b}-\omega_{a b}\right)=2 i H_{b c}^{\hat{a}} \theta^{c} .
$$

Симметризуя и альтернируя это соотношение по индексам $a$ и $b$, получим соответственно

$$
\omega_{b}^{a}+\omega_{a}^{b}=2 i H_{(a b) c} \theta^{c}, \quad \omega^{a b}-\omega_{a b}=2 i H_{[a b] c} \theta^{c},
$$

где $H_{a b c}=H_{b c}^{\hat{a}}$. Переписав теперь (34) в форме

$$
\theta_{b}^{a}=\frac{1}{2}\left(\omega_{b}^{a}-\omega_{a}^{b}\right)+\frac{1}{2}\left(\omega^{a b}+\omega_{a b}\right),
$$

с учетом соотношений $(35),(17),(22)$ и (25) получим

$$
\omega_{b}^{a}=\theta_{b}^{a}-\sqrt{2} \widetilde{B}^{c a b} \theta_{c}+i H_{a b c} \theta^{c}
$$

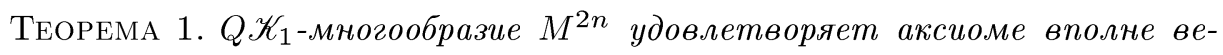
щественных подмногообразий тогда и только тогда, когда оно является почти келеровым многообразием. 
ДокАЗАТЕЛЬСТво. Фиксируем точку $p_{0} \in M$ и $n$-мерное вполне вешественное подпространство $L \subset T_{p_{0}}(M)$. Пусть $N \subset M$ - вполне вешественное подмногообразие, проходящее через эту точку в направлении $L$. Это означает, что $p_{0} \in N, T_{p_{0}}(N)=L$. Пусть $\left(p, e_{1}, \ldots, e_{n}\right)$ - ортонормированный репер пространства $T_{p}(N), \quad p \in N$, и $\left(p, \theta^{1}, \ldots, \theta^{n}\right)$ - дуальный корепер. Как уже отмечалось, они порождают вещественно-адаптированный репер и дуальный ему корепер пространства $T_{p}(M)$ соответственно. Пусть $\left(p, \varepsilon_{1}, \ldots, \varepsilon_{n}, \varepsilon_{\hat{1}}, \ldots, \varepsilon_{\hat{n}}\right)$ и $\left(p, \omega^{1}, \ldots, \omega^{n}, \omega_{1}, \ldots, \omega_{n}\right)$ - соответствуюшие им $A$-репер и дуальный ему корепер. Согласно (22) подмногообразие $N \subset M$ задается системой Пфафффа $\theta^{\hat{a}}=0$, и в силу (25)

$$
\omega^{a}=\frac{1}{\sqrt{2}} \theta^{a}, \quad \omega_{a}=\frac{1}{\sqrt{2}} \theta^{a}
$$

(для простоты сужение формы на $N$ обозначаем той же буквой, что и саму форму). Систему (37) можно рассматривать как систему Пфаффа подмногообразия $N$. Найдем условия ее интегрируемости. Продифференцируем внешним образом первое равенство в (37):

$$
d \omega^{a}=\frac{1}{\sqrt{2}} d \theta^{a} .
$$

С учетом первого соотношения из $(9),(27)$ и (22) это равенство примет вид

$$
\sqrt{2}\left(\omega_{b}^{a} \wedge \omega^{b}+B^{a b c} \omega_{b} \wedge \omega_{c}\right)=\theta_{b}^{a} \wedge \theta^{b},
$$

или в силу (37)

$$
\left(\omega_{b}^{a}-\theta_{b}^{a}\right) \wedge \theta^{b}+\frac{1}{\sqrt{2}} B^{a b c} \theta_{b} \wedge \theta_{c}=0 .
$$

Подставляя сюда (36), с учетом свойств симметрии второй квадратичной формы и линейной независимости базисных форм получим

$$
2 \widetilde{B}^{[b|a| c]}-B^{a b c}=0,
$$

что, очевидно, равносильно соотношению $B^{[a b c]}=0$ и в силу предложения 1 равносильно тому, что $M$ - почти келерово многообразие. Аналогично, с учетом второго соотношения в (9) и того обстоятельства, что в ортонормированном репере $\theta_{b}^{a}+\theta_{a}^{b}=0$, проверяется, что условие $d \omega_{a}=\frac{1}{\sqrt{2}} d \theta^{a}$ вьполняется тождественно в силу (37). Таким образом, система (37) вполне интегрируема тогда и только тогда, когда $M$ - почти келерово многообразие. В этом и только этом случае через каждую точку $r$ пространства расслоения $A$-реперов, а значит, и пространства вещественно-адаптированных реперов над $M$ проходит единственное интегральное многообразие максимальной размерности. Его проекция на $M$ является $n$-мерным подмногообразием многообразия $M$, которое в силу (37) является вполне вещественным подмногообразием, касательное пространство которого в начале вешественно-адаптированного репера $r$ совпадает с линейной оболочкой первых $n$ векторов этого репера. В силу произвола в выборе $r$ отсюда следует утверждение теоремы.

В качестве важного следствия доказанной теоремы получаем следующий результат. 
ТЕОРема 2. Через каждую точку р симплектического многообразия $(M, \Omega)$ в направлении любой лагранжевой плоскости $L \subset T_{p}(M)$ проходит подмногообразие Лагранжса.

ДокАЗАТЕЛЬСТво. В силу замечания 1 утверждение теоремы 2 равносильно справедливости аксиомы вполне вещественных подмногообразий для многообразия $M$ как $A \mathscr{K}_{1}$-многообразия.

ЗАмЕчАниЕ 3 . Подмногообразие Лагранжа, проходящее через данную точку $p$ в направлении данной лагранжевой плоскости $L \subset T_{p}(M)$, вообще говоря, не единственно. Рассмотрим, например, стандартную симплектическую структуру на пространстве $\mathbb{R}^{2}$, заданную 2 -формой $\Omega=d x^{1} \wedge d x^{2}$. Непосредственно проверяется, что подмногообразие $N_{1} \subset \mathbb{R}^{2}$, заданное уравнением $x^{2}=\left(x^{1}\right)^{2}$, равно как и подмногообразие $N_{2} \subset \mathbb{R}^{2}$, заданное уравнением $x^{2}=\left(x^{1}\right)^{3}$, являются одномерными подмногообразиями Лагранжа, проходящими через точку $O(0,0,0)$. Очевидно, их касательные пространства в точке $O$ совпадают и задаются уравнением $x^{2}=0$. Рассмотрим стандартную симплектическую структуру на пространстве $\mathbb{R}^{2 n}$, заданную 2-формой

$$
\Omega=\sum_{i=1}^{n} d x^{i} \wedge d x^{n+i}
$$

Рассмотрим произвольную гладкую функцию $y=f\left(x^{1}, \ldots, x^{n}\right)$. Построим $n$ функций

$$
x^{n+i}=\frac{\partial f}{\partial x^{i}}, \quad i=1, \ldots, n .
$$

Рассмотрим $n$-мерное подмногообразие $N \subset \mathbb{R}^{2 n}$, заданное этой системой уравнений. Очевидно, оно является подмногообразием Лагранжа. Если фиксировать точку $p_{0}=\left(x_{0}^{1}, \ldots, x_{0}^{n}\right) \in N$, то касательное пространство к $N$ в этой точке задается уравнением

$$
d x^{n+i}=\left.\frac{\partial^{2} f}{\partial x^{i} \partial x^{j}}\right|_{p=p_{0}} d x^{j}, \quad i=1, \ldots, n .
$$

В частности, если $f_{1}$ и $f_{2}$ - две функции, имеюшие одинаковый гессиан в точке $p_{0}$, то они порождают, вообще говоря, различные подмногообразия Лагранжа, имеющие одинаковый интегральный элемент в этой точке.

TЕорема 3. Q $\mathscr{K}_{1}$-многообразие размерности выше четырех удовлетворяет аксиоме вполне вещественных плоскостей тогда и только тогда, когда оно является натуральной пространственной формой и, следовательно, локально әквивалентно одному из следующих многообразий:

1) при $c \neq 0$ - многообразию $\mathbb{R} P_{n} \diamond \mathbb{R} P_{n}$ невырожденных нуль-пар вещественного проективного пространства;

2) при с $=0$ - двойному евклидову пространству $\mathbb{R}^{n} \otimes \mathbb{R}^{n}$. 
ДоказАТЕльСтво. Необходимость немедленно вытекает из [2, теорема 38]. Обратно, пусть $M^{2 n}$ - натуральная пространственная форма. Надо доказать, что через каждую точку многообразия $M^{2 n}$ в любом $n$-мерном вполне вещественном направлении проходит единственное вполне геодезическое $n$-мерное интегральное многообразие. Заметим, что вполне геодезичность интегрального многообразия равносильна обращению в нуль его второй квадратичной формы [3], а потому требование вполне геодезичности интегральных многообразий системы (37) равносильно добавлению к этой системе уравнений (36) при $H_{a b c}=0$, которые мы запишем в форме

$$
\theta_{b}^{a}=\omega_{b}^{a} .
$$

Условие интегрируемости полученной системы Пфаффа задается соотношением

$$
d \theta_{b}^{a}=d \omega_{b}^{a},
$$

или, с учетом (15),

$$
\theta_{c}^{a} \wedge \theta_{b}^{c}+\frac{1}{2} \widetilde{R}_{b c d}^{a} \theta^{c} \wedge \theta^{d}=\omega_{c}^{a} \wedge \omega_{b}^{c}+\omega_{\hat{c}}^{a} \wedge \omega_{b}^{\hat{c}}+\frac{1}{2} R_{b i j}^{a} \omega^{i} \wedge \omega^{j}
$$

где $\widetilde{R}_{b c d}^{a}$ - компоненты тензора Римана-Кристоффеля псевдориманова многообразия $N$. Поскольку $M-\mathscr{K}_{1}$-многообразие, $\omega_{b}^{\hat{a}}=\omega_{\hat{b}}^{a}=0$ в силу (17). Кроме того, заметим, что ввиду вполне геодезичности подмногообразия $N \subset M$ имеем $\widetilde{R}=\left.R\right|_{N}$ и, значит,

$$
\widetilde{R}_{b c d}^{a} \theta^{c} \wedge \theta^{d}=C_{i}^{a} D_{b}^{j} D_{c}^{k} D_{d}^{l} R_{j k l}^{i} \theta^{c} \wedge \theta^{d}=C_{i}^{a} D_{b}^{j} R_{j k l}^{i} \omega^{k} \wedge \omega^{l} .
$$

Подставляя эти соотношения в (40), после необходимых сокращений с учетом (17), (39) и линейной независимости базисных форм получим

$$
C_{i}^{a} D_{b}^{j} R_{j c d}^{i}=R_{b c d}^{a}+R_{b c \hat{d}}^{a}+R_{b c \hat{d}}^{a}+R_{b \hat{c} \hat{d}}^{a} .
$$

$\mathrm{C}$ учетом (23) и (24) это соотношение можно переписать в форме

$$
\begin{aligned}
& -R_{\hat{a} b c d}-R_{\hat{a} b c \hat{d}}-R_{\hat{a} b \hat{c} d}-R_{a b \hat{c} \hat{d}}+R_{a b c d}+R_{a b c \hat{d}}+R_{a b \hat{c} d}+R_{a b \hat{c} \hat{d}} \\
& \quad+R_{\hat{a} \hat{b} c d}+R_{\hat{a} \hat{b} c \hat{d}}+R_{\hat{a} \hat{b} \hat{c} d}+R_{\hat{a} \hat{b} \hat{c} \hat{d}}+R_{a \hat{b} c d}+R_{a \hat{b} c \hat{d}}+R_{a \hat{b} \hat{c} d}+R_{a \hat{b} \hat{c} \hat{d}}=0 .
\end{aligned}
$$

С учетом (16) и (18) после необходимых сокращений окончательно получаем условия интегрируемости системы (37), (39):

$$
A_{b c}^{a d}-A_{b d}^{a c}+A_{a c}^{b d}-A_{a d}^{b c}=0 .
$$

Очевидно, на натуральной пространственной форме эти условия выполняются тождественно в силу предложений 1 и 3 . Таким образом, система (37), (39) вполне интегрируема. Повторяя то же рассуждение, что и при окончании доказательства теоремы 1 , получаем, что через каждую точку $p$ многообразия $M$ в направлении любого вполне вешественного подпространства $L \subset T_{p}(M)$ проходит $n$-мерное вполне геодезическое вполне вешественное подмногообразие. 
ОПРЕДЕЛЕНИЕ $3 . n$-мерное вполне геодезическое подмногообразие Лагранжа симплектического многообразия $\left(M^{2 n}, \Omega\right)$ назовем строго лагранжевы. (короче, $s$-лагранжевым).

Из теоремы 3 немедленно следует

ТЕОРема 4. Через каждую точку симплектического многообразия $\left(M^{2 n}, \Omega\right), \quad n>2$, в направлении любой $n$-мерной лагранжевой плоскости в этой точке проходит единственное s-лагранжево подмногообразие тогда и только тогда, когда многообразие $M^{2 n}$ является натуральной пространственной формой.

ЗАмечАнИЕ 4. Из доказательства теоремы 3 следует, что для натуральных пространственных форм утверждения теорем 3 и 4 справедливы без каких-либо ограничений на их размерность.

Пусть $N \subset M^{2 n}-s$-лагранжево подмногообразие $A \mathscr{K}_{1}$-многообразия. В этом случае (36) запишется в форме

$$
\omega_{b}^{a}=\theta_{b}^{a}-\sqrt{2} B^{c a b} \theta_{c} .
$$

Выпишем сужение второй группы структурных уравнений (12) $A \mathscr{K}_{1}$-структуры на подмногообразие $N$. Согласно (42) имеем

$$
\begin{gathered}
d \theta_{b}^{a}-2 d B^{c a b} \wedge \omega_{c}-2 B^{c a b} d \omega_{c}=\left(\theta_{c}^{a}-2 B^{h a c} \omega_{h}\right) \wedge\left(\theta_{b}^{c}-2 B^{r c b} \omega_{r}\right) \\
+\left(2 B^{a d h} B_{h b c}+A_{b c}^{a d}\right) \omega^{c} \wedge \omega_{d}+B_{b c d}^{a} \omega^{c} \wedge \omega^{d}-B_{b}^{a c d} \omega_{c} \wedge \omega_{d} .
\end{gathered}
$$

Подставляя сюда второе соотношение из (9) и (14), с учетом (37) после необходимых сокрашений получим

$$
\begin{aligned}
d \theta_{b}^{a}= & \theta_{c}^{a} \wedge \theta_{b}^{c}+\left\{\left(B^{h a g} B_{r b g}-B^{r a g} B_{h b g}\right)\right. \\
& +\frac{1}{4}\left(2 B^{a r g} B_{g b h}-2 B^{a h g} B_{g b r}+A_{b h}^{a r}-A_{b r}^{a h}\right) \\
& \left.+\frac{1}{2}\left(B_{b h r}^{a}-B_{b}^{a h r}+B_{h}^{r a b}-B_{r}^{h a b}+B^{r a b h}-B^{h a b r}\right)+B^{c a b} B_{c h r}\right\} \theta^{h} \wedge \theta^{r} .
\end{aligned}
$$

С другой стороны, с учетом соотношений $(27),(32)$ и вполне геодезичности подмногообразия имеем

$$
d \theta_{b}^{a}=\theta_{c}^{a} \wedge \theta_{b}^{c}+\frac{1}{2} \widetilde{R}_{b h r}^{a} \theta^{h} \wedge \theta^{r} .
$$

Следовательно, в ортонормированном репере подмногообразия $N$

$$
\begin{aligned}
\widetilde{R}_{b c d}^{a}= & 2 B^{c a g} B_{d b g}-2 B^{d a g} B_{c b g} \\
& +\frac{1}{2}\left(2 B^{a d g} B_{g b c}-2 B^{a c g} B_{g b d}+A_{b c}^{a d}-A_{b d}^{a c}\right) \\
& +B_{b c d}^{a}-B_{b}^{a c d}+B_{c}^{d a b}-B_{d}^{c a b}+B^{d a b c}-B^{c a b d}+2 B^{h a b} B_{h c d}
\end{aligned}
$$


Пусть, в частности, $M^{2 n}-A \mathscr{H}_{1}$-многообразие точечно постоянной голоморфной секционной кривизны $c$. Тогда, подставляя (21) в (43), после упрощений с учетом предложения 1 получим

$$
\begin{aligned}
\widetilde{R}_{b f d}^{a}= & -\frac{c}{4}\left(\delta_{f}^{a} \delta_{b}^{d}-\delta_{d}^{a} \delta_{b}^{f}\right)+B^{h a f} B_{h b d}-B^{h a d} B_{h b f}+2 B^{h a b} B_{h f d} \\
& +B_{b f d}^{a}-B_{b}^{a f d}+B_{f}^{d a b}-B_{d}^{f a b}+B^{d a b f}-B^{f a b d} .
\end{aligned}
$$

В частности, пусть $M$ - натуральная пространственная форма голоморфной секционной кривизны $c$. Тогда в силу предложения 1 и соотношений (14) получаем

$$
B^{a b f}=B_{d}^{a b f}=B^{a b f d}=B_{a b f}=B_{a b f}^{d}=B_{a b f d}=0
$$

и, значит,

$$
\widetilde{R}_{b f d}^{a}=-\frac{c}{4}\left(\delta_{f}^{a} \delta_{b}^{d}-\delta_{d}^{a} \delta_{b}^{f}\right),
$$

т. е. $N$ - пространство постоянной кривизны $\frac{c}{4}$. Доказана

ТЕОРемА 5. s-лагранжевы подмногообразия натуральной пространственной формы голоморфной секционной кривизны с являются пространствами постоянной кривизны $\frac{c}{4}$.

1.3. Приложения к геометрии главных $T^{1}$-расслоений. Хорошо известно, что на нечетномерном многообразии $M^{2 n+1}$ аналогом $A \mathscr{K}_{1}$-структуры является контактная метрическая структура гиперболического типа (C $\mathscr{K}_{1}$-структура), т. е. совокупность $(\xi, \eta, \Phi, g)$, где $\xi$ - вектор, $\eta$ - 1-форма максимального ранга, называемая контактной формой, или контактной структурой, $\Phi$ - тензор типа $(1,1)$ (структурный эндоморфизм), $g=\langle\cdot, \cdot\rangle-$ псевдориманова метрика, причем

$$
\begin{gathered}
\eta(\xi)=1, \quad \Phi(\xi)=0, \quad \eta \circ \Phi=0, \quad d \eta(X, Y)=\Omega(X, Y), \\
\Phi^{2}=\mathrm{id}-\eta \otimes \xi, \quad\langle\Phi X, \Phi Y\rangle=-\langle X, Y\rangle+\eta(X) \eta(Y) ;
\end{gathered}
$$

здесь $X, Y \in \mathfrak{X}(M)$ и $\Omega(X, Y)=\langle X, \Phi Y\rangle$ - 2-форма на $M$, называемая фундаментальной формой структуры. Если $\eta$ - форма Киллинга, С $\mathscr{K}_{1}$-структуру по аналогии с классической терминологией [9] назовем $K$-контактной. Хорошо известно также [9], что контактное распределение $\operatorname{ker} \eta$ допускает $n$-мерные интегральные многообразия, называемые подмногообразиями Лежандра, и не допускает интегральных многообразий большей размерности. Эти подмногообразия являются естественным аналогом подмногообразий Лагранжа в симплектической геометрии. В частности, такими же рассуждениями, как и для подмногообразий Лагранжа, доказывается, что через каждую точку $p$ контактного многообразия $M^{2 n+1}$ в направлении любого подпространства Лежандра (т. е. $n$-мерного подпространства, аннулируюшего контактную форму и ее внешний дифференциал) $L \subset T_{p}(M)$ проходит подмногообразие Лежандра (это свойство мы называем noлуинтегрируемостью контактного распределения). 
Как и в случае симплектических многообразий, доказывается, что подмногообразие Лежандра с начальными данными $(p, L)$, вообще говоря, не единственно, однако если $\operatorname{dim} M>5$, то совокупность таких подмногообразий при любых начальных данных в случае, если метрическое расширение исходной контактной структуры является $K$-контактной структурой, содержит единственное вполне геодезическое подмногообразие (это свойство мы называем геодезической интегрируемостью контактного распределения) тогда и только тогда, когда это расширение является сасакиевой структурой гиперболического типа постоянной $\Phi$-голоморфной секционной кривизны (т. е. постоянной кривизны в двумерных направлениях вида $X \wedge \Phi X, X \in \operatorname{ker} \eta)$. Многообразия, наделенные такой структурой, называются сасакиевыми пространственными формами гиперболического типа. Их полная классификация приведена в [4]. Легко видеть, что контактное распределение любой сасакиевой пространственной формы гиперболического типа геодезически интегрируемо.

Вполне геодезические подмногообразия Лежандра назовем подмногообразиями Блэра. Подмногообразия Блэра являются, таким образом, контактным аналогом $s$-лагранжевых подмногообразий симплектического многообразия. Как и для $s$-лагранжевых подмногообразий, проверяется, что подмногообразия Блэра сасакиевой пространственной формы гиперболического типа $\Phi$-голоморфной секционной кривизны $c$ являются пространствами постоянной кривизны $\frac{c-3}{4}$.

Укажем на одну замечательную связь между этими видами подмногообразий. Пусть $M^{2 n}-A \mathscr{K}_{1}$-многообразие с целочисленной фундаментальной формой $\Omega$ (назовем соответствующую $А \mathscr{K}_{1}$-структуру обобщенной структурой Ходжа). Класс когомологий этой формы является характеристическим классом главного $T^{1}$-расслоения $\pi: P \rightarrow M$ над $M$ (расслоения Бутби-Вана [9]). Это главное расслоение допускает каноническую связность с формой $\omega$ такой, что

$$
d \omega=\pi^{*} \Omega
$$

(см. [10]). Очевидно, форма $\omega$ является контактной структурой на многообразии $P$, которую назовем канонической. Вместе с естественным лифтом исходной $A \mathscr{K}_{1}$-структуры в пространство расслоения она, очевидно, определяет $C \mathscr{K}_{1}$-структуру на многообразии $P$, которую назовем горизонтальным. поднятием исходной $A \mathscr{K}_{1}$-структуры. Очевидно, контактное распределение этой $C \mathscr{K}_{1}$-структуры совпадает с горизонтальным распределением канонической связности, а ее фундаментальная форма - с формой $\pi^{*} \Omega$. Более того, легко видеть, что производная Ли индуцированного на $P$ метрического тензора в направлении вектора, дуального контактной форме, равна нулю, т. е. контактная форма является формой Киллинга, а индуцированная на $P$ контактная метрическая структура $-K$-контактной структурой.

ТЕОРема 6. Горизонтальное распределение канонической связности в расслоении Бутби-Вана над обобщенным многообразием Ходжа полуинтегрируeм⿱宀⿻. 
Естественно называть интегральные подмногообразия горизонтального распределения горизонтальными подмногообразиями. Эти подмногообразия есть не что иное, как подмногообразия Лежандра относительно канонической контактной структуры на многообразии $P$. Из (44) немедленно следует, что проекция $N=\pi(\widetilde{N})$ горизонтального подмногообразия $\widetilde{N}$ на базу расслоения Бутби-Вана является подмногообразием Лагранжа исходного симплектического многообразия. В таком случае будем называть подмногообразие Лежандра $\widetilde{N}$ горизонтальны.м поднятием подмногообразия Лагранжа $N$.

Нетрудно проверить, что обобщенное многообразие Ходжа $M$ будет комплексной пространственной формой голоморфной секционной кривизны $c$ тогда и только тогда, когда пространство расслоения Бутби-Вана над ним будет сасакиевой пространственной формой $\Phi$-голоморфной секционной кривизны $c+3$. С учетом сделанных замечаний верна

ТЕОРема 7. Горизонтальное распределение канонической связности в расслоении Бутби-Вана над обобщенным многообразием Ходжса $М$ размерности выше четырех геодезически интегрируемо тогда и только тогда, когда М натуральная пространственная форма.

Пусть $N \subset P$ - подмногообразие Блэра. Из определения метрики, индуцированной в пространстве расслоения Бутби-Вана, с учетом рассуждений, приведенных в доказательстве теоремы 1, вполне идентичных как в симплектическом, так и в контактном случаях, убеждаемся, что, во-первых, сужение естественной проекции $\pi$ на подмногообразие Лежандра является (локальной) изометрией и, во-вторых, это отображение вполне геодезические подмногообразия Лежандра переводит во вполне геодезические подмногообразия Лагранжа, т. е. подмногообразия Блэра - в $s$-лагранжевы подмногообразия. Таким образом, подмногообразия Блэра являются горизонтальными поднятиями $s$-лагранжевых подмногообразий. Более того, из определения горизонтального поднятия обобщенной структуры Ходжа следует, что соответствующая $K$-контактная структура инвариантна относительно действия структурной групшы главного $T^{1}$-расслоения, которое, таким образом, подмногообразия Лежандра переводит в подмногообразия Лежандра, а подмногообразия Блэра - в подмногообразия Блэра.

Пусть $N \subset M-s$-лагранжево подмногообразие натуральной пространственной формы, $p \in N$ - произвольная точка, $q \in \pi^{-1}(p), L \subset T_{q}(P)$ - горизонтальный подъем пространства $T_{p}(N), \mathscr{P} \subset P$ - максимальное подмногообразие Блэра с начальными данными $(q, L)$. Рассмотрим подгруппу $G$ структурной груп-

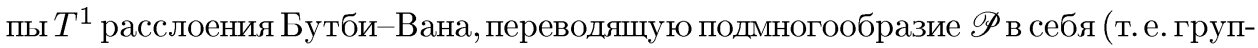
пу инвариантности этого подмногообразия). Очевидно, это собственная и, значит, дискретная подгруппа. В силу компактности $T^{1}$ эта подгруппа конечна. Орбита каждой точки из $\mathscr{P}$ относительно нее есть не что иное, как пересечение полного прообраза проекции этой точки на базу с $\mathscr{P}$. Пусть $U-$ окрестность точки $q$ в $\mathscr{P}$, сужение на которую отображения $\pi$ является изометрией. Поскольку $\pi$ - открытое отображение, подмногообразие $\widetilde{N}=\pi(\mathscr{P}) \subset M$ является $s$-лагранжевым подмногообразием с начальными данными $\left(p, T_{p}(N)\right)$, определенным в окрестности $\pi(U)$ 
точки $p$. В силу теоремы 4 это подмногообразие совпадает с $N$ в некоторой окрестности $W$ точки $p$. Рассмотрим $\pi^{-1}(W) \cap \mathscr{P}$. В соответствии со сказанным

$$
\pi^{-1}(W) \cap \mathscr{P}=\bigcup_{i=1}^{n} W_{i},
$$

где $n$ - число элементов подгруппы $G$, причем $W_{i}$ переводится в $W_{j}$ действием соответствующей изометрии из $G$, а $\pi\left(W_{i}\right)=W, i, j=1, \ldots, n$. Ввиду произвольности выбора $p \in N$ отображение $\pi: P \rightarrow M$ индуцирует накрытие $\varpi: \mathcal{N} \rightarrow N$, где $\mathscr{N}=\pi^{-1}(N) \cap \mathscr{P}$ - подмногообразие Блэра, которое назовем полнылм горизонтальным поднятием $s$-лагранжева подмногообразия $N, \varpi=\left.\pi\right|_{\mathcal{N}}$. Таким образом, доказана

ТЕОРема 8. Каждое $s$-лагранжево подмногообразие $N$ натуральной пространственной формы $M$ конечнолистно накрывается некоторым подмногообразием Бләра в расслоении Бутби-Вана над $М$, являющимся полным горизонтальным поднятием подмногообразия $N$. При этом группа инвариантности подмногообразия Бләра является подгруппой фундаментальной группь соответствующего $s$-лагранжева подмногообразия.

СлЕДСТВИЕ 1. Каждое односвязное s-лагранжево подмногообразие $N$ натуральной пространственной формы $M$ изометрично некоторому подмногообразию Блэра с тривиальной группой инвариантности в расслоении Бутби-Вана над $M$, являющемуся полным горизонтальным поднятием подмногообразия $N$.

\section{§ 2. Дифференциальная геометрия многообразия нуль-пар}

Многообразия нуль-пар представляют собой важнейшие примеры $A \mathscr{H}_{1}$-многообразий. Мы уже подробно обсуждали этот вопрос во введении. Кроме указанных там причин, эти многообразия интересны тем, что они позволяют моделировать и конструктивно исследовать механизм важнейших особенностей геометрии

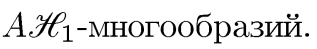

Невырожсденную нуль-пару в вещественном проективном пространстве $\mathbb{R} P^{n}$ составляют точка и неинцидентная ей гиперплоскость. Совокупность всех таких нуль-пар можно естественным образом наделить структурой $2 n$-мерного, связного, ориентированного, некомпактного, вещественно-аналитического многообразия $\mathrm{GL}(n+1, \mathbb{R}) / \mathrm{GL}(1, \mathbb{R}) \times \mathrm{GL}(n, \mathbb{R})$, на котором внутренним образом определяются структура прямого произведения, симплектическая структура и псевдориманова метрика нейтральной сигнатуры. Оказывается, что многообразие невырожденных нуль-пар несет каноническую келерову структуру гиперболического типа постоянной голоморфной секционной кривизны $c \neq 0$. Таким образом, многообразие невырожденных нуль-пар вещественного проективного пространства является гиперболическим аналогом комплексных пространственных форм в эрмитовой 
геометрии. При этом аналогом вполне вещественных подмногообразий на многообразии нуль-пар являются подмногообразия, аннулирующие 2-форму канонической симплектической структуры, т. е лагранжевы подмногообразия.

2.1. Гладкая структура на многообразии нуль-пар. Пусть $P_{n}=P(W)-$ вешественное проективное пространство размерности $n$, порожденное векторным пространством $W$. Пространство $P_{n}$ обладает структурой $n$-мерного вещественно-аналитического многообразия относительно гладкости, заданной при помоши аффинных карт. Пространство $P_{n}$ связно. Каноническая проекция $p: W \backslash 0 \rightarrow$ $P(W)$ является аналитическим отображением и называется отображсением проективизащии. Если вектор $a \in W$ порождает точку $A \in P(W)$, т.е. $p(a)=A$, то точку $A$ будем отождествлять с одномерным векторным подпространством $\langle a\rangle$, натянутым на вектор $a$. Итак, $A=p(a)=\langle a\rangle$.

Рассмотрим короткую последовательность векторных пространств и их гомоморфизмов

$$
\mathscr{O} \rightarrow\langle a\rangle \stackrel{i}{\rightarrow} T_{a} W \equiv W \stackrel{\left(p_{*}\right)_{a}}{\longrightarrow} T_{A} P(W) \rightarrow \mathscr{O}
$$

где $i$ - гомоморфизм включения. Легко показать, что рассматриваемая последовательность является точной, т.е. $\operatorname{Im} i=\operatorname{ker}\left(p_{*}\right)_{a}=\langle a\rangle$. Следовательно, существует естественный изоморфизм $\cong$ между касательным векторным пространством $T_{A} P(W)$ в точке $A$ проективного пространства $P(W)$ и факторпространством $W /\langle a\rangle$ пространства $W$ по одномерному подпространству $\langle a\rangle$. Таким образом, $T_{A} P(W) \cong W /\langle a\rangle$. С точностью до этого изоморфизма имеет место равенство

$$
\left(p_{*}\right)_{a} b=\langle a\rangle+b \quad \forall b \in W \equiv T_{a} W .
$$

Пусть теперь $W^{*}$ - векторное пространство, сопряженное для $W$. Тогда $P_{n}^{*}=$ $P\left(W^{*}\right)$ - проективное пространство, двойственное для $P_{n}$, т. е. пространство гиперплоскостей в $P_{n}$. Если $\xi \in W^{*}$, то будем использовать отождествления $\Xi=$ $p(\xi)=\langle\xi\rangle, T_{\Xi} P\left(W^{*}\right) \cong W^{*} /\langle\xi\rangle$ и $\left(p_{*}\right)_{\xi} \eta=\langle\xi\rangle+\eta \forall \eta \in W^{*} \equiv T_{a} W^{*}$.

ОПРЕДЕЛЕНИЕ 4. Нуль-nарой в $P_{n}$ называется пара $(A, \Xi)$, где $A \in P^{n}$ и $\Xi \in$ $P_{n}^{*}$. Нуль-пара называется вырожденной, если точка $A=\langle a\rangle$ и гиперплоскость $\Xi=\langle\xi\rangle$ иншидентны, т.е. $A \in \Xi$ (или, что эквивалентно, $\xi(a)=0$ ). В противном случае нуль-пара называется невырожденной.

Обозначим через $M$ множество всех нуль-пар. Ясно, что $M=P_{n} \times P_{n}^{*}$ является $2 n$-мерным вещественно-аналитическим многообразием относительно структуры прямого произведения аналитических многообразий $P_{n}$ и $P_{n}^{*}$. Многообразие $M$ связно и компактно.

Пусть $M_{0}$ - подмножество в $M$, состоящее из всех вырожденных нуль-пар. Фиксируем базис $\left\{e_{u}\right\}$ векторного пространства $W$, и пусть $\left\{e^{u}\right\}$ - сопряженньй кобазис. Если $a=a^{u} e_{u}$ - ненулевой вектор, то $\left(a^{0}, a^{1}, \ldots, a^{n}\right)$ - однородные координаты точки $A=p(a) \in P_{n}$. Если же $\xi=\xi_{u} e^{u}-$ ненулевой ковектор, то $\left(\xi_{0}, \xi_{1}, \ldots, \xi_{n}\right)$ - однородные координаты гиперплоскости $\Xi=p(\xi) \in P_{n}^{*}$ (или, что 
то же самое, ее тангенциальные координаты). Упорядоченный набор из $2 n+2$ чисел $\left(a^{0}, a^{1}, \ldots, a^{n} ; \xi_{0}, \xi_{1}, \ldots, \xi_{n}\right)$ называется однородными координатами нуль$n a p b l(A, \Xi)$. В однородных координатах условие вырожденности нуль-пары $(A, \Xi)$ имеет вид

$$
a^{0} \xi_{0}+a^{1} \xi_{1}+\cdots+a^{n} \xi_{n}=0
$$

Это уравнение определяет $M_{0}$ как гладкую гиперповерхность в $M$. Ясно, что $M_{0}$ замкнуто в $M$.

Пусть $\mathfrak{N}=M \backslash M_{0}-$ совокупность всех невырожденных нуль-пар. Ясно, что $\mathfrak{N}-$ открытое подмногообразие в $M$.

ПРЕДЛОЖЕНИЕ 4. Многообразие $\mathfrak{N}$ всех невырождденных нуль-пар линейно связно.

ДокАЗАТЕЛЬСТво. Необходимо доказать, что для любых двух невырожденных нуль-пар $\left(A_{1}, \Xi_{1}\right)$ и $\left(A_{2}, \Xi_{2}\right)$ сушествует непрерывная кривая в $\mathfrak{N}$, соединяюшая $\left(A_{1}, \Xi_{1}\right)$ с $\left(A_{2}, \Xi_{2}\right)$.

1. Прежде всего докажем, что любые две невырожденные нуль-пары вида $(A, \Xi)$ и $\left(A^{\prime}, \Xi\right)$ принадлежат одной компоненте линейной связности. Прямая $A A^{\prime} \subset P_{n}$ гомеоморфна окружности и разбивается точками $A$ и $A^{\prime}$ на пару отрезков, лишь одному из которых принадлежит точка пересечения гиперплоскости $\Xi$ с прямой $A A^{\prime}$. Пусть $L_{A A^{\prime}}$ - отрезок, соединяющий $A$ и $A^{\prime}$ и не имеющий общих точек с $\Xi$. Тогда кривая $L_{A A^{\prime}} \times \Xi$ содержится в $\mathfrak{N}$ и соединяет $(A, \Xi)$ с $\left(A^{\prime}, \Xi\right)$. Обозначим ее $(A, \Xi) \rightarrow\left(A^{\prime}, \Xi\right)$.

2. В силу принципа двойственности из п. 1 следует, что любые две невырожденные нуль-пары вида $(A, \Xi)$ и $\left(A, \Xi^{\prime}\right)$ также принадлежат одной компоненте линейной связности.

3. Для любых двух невырожденных нуль-пар $\left(A_{1}, \Xi_{1}\right)$ и $\left(A_{2}, \Xi_{2}\right)$ существует точка $A_{0}$, которая неинцидентна гиперплоскостям $\Xi_{1} \Xi_{2}$. Поэтому в силу пп. 1 и 2 сушествует непрерывная кривая

$$
\left(A_{1}, \Xi_{1}\right) \rightarrow\left(A_{0}, \Xi_{1}\right) \rightarrow\left(A_{0}, \Xi_{2}\right) \rightarrow\left(A_{2}, \Xi_{2}\right)
$$

которая соединяет $\left(A_{1}, \Xi_{1}\right)$ с $\left(A_{2}, \Xi_{2}\right)$ и содержится в $\mathfrak{N}$.

Рассмотрим два отображения

$$
\Psi, \Omega:\left(W \times W^{*}\right) \times\left(W \times W^{*}\right) \times\left(W \times W^{*}\right) \rightarrow \mathbb{R},
$$

определенные соотношениями

$$
\begin{aligned}
& \Psi(a \times \xi ; b \times \eta ; c \times \zeta)=-\frac{1}{\xi(a)}\left(\begin{array}{cc}
\xi(a) & \xi(c) \\
\eta(a) & \eta(c)
\end{array}|+| \begin{array}{ll}
\xi(a) & \xi(b) \\
\zeta(a) & \zeta(b)
\end{array} \mid\right), \\
& \Omega(a \times \xi ; b \times \eta ; c \times \zeta)=\frac{1}{\xi(a)}\left(\begin{array}{cc}
\xi(a) & \xi(c) \\
\eta(a) & \eta(c)
\end{array}|-| \begin{array}{cc}
\xi(a) & \xi(b) \\
\zeta(a) & \zeta(b)
\end{array} \mid\right),
\end{aligned}
$$


которые имеют смысл всюду, где $\xi(a) \neq 0$. Формулы $(45),(46)$ линейны по двум последним аргументам и инвариантны относительно преобразований вида

$$
a \mapsto \lambda a, \quad \xi \mapsto \mu \xi ; \quad b \mapsto b+\langle a\rangle, \quad \eta \mapsto \eta+\langle\xi\rangle ; \quad c \mapsto c+\langle a\rangle, \quad \zeta \mapsto \zeta+\langle\xi\rangle
$$

Поэтому формулы (45) и (46) можно интерпретировать как отображения

$$
\Psi, \Omega: \bigcup_{\substack{a \in W \backslash 0 \\ \xi \in W^{*} \backslash 0 \\ \xi(a) \neq 0}}\left((\langle a\rangle \times\langle\xi\rangle) \times\left(W /\langle a\rangle \oplus W^{*} /\langle\xi\rangle\right) \times\left(W /\langle a\rangle \oplus W^{*} /\langle\xi\rangle\right)\right) \rightarrow \mathbb{R}
$$

т. е. $\Psi$ и $\Omega$ - билинейные формы на многообразии невырожденных нуль-пар $\mathfrak{N}$. При этом форма $\Psi$ симметрична, а форма $\Omega$ кососимметрична. Если

$$
\begin{gathered}
a \in W \backslash 0, \quad \xi \in W^{*} \backslash 0, \quad x=p(a) \times p(\xi) \in \mathfrak{N}, \\
b, c \in W \equiv T_{a} W, \quad \eta, \zeta \in W^{*} \equiv T_{\xi} W^{*}, \\
X=\left(p_{*}\right)_{a} b \oplus\left(p_{*}\right)_{\xi} \eta \in T_{x} \mathfrak{N}, \quad Y=\left(p_{*}\right)_{a} c \oplus\left(p_{*}\right)_{\xi} \zeta \in T_{x} \mathfrak{N},
\end{gathered}
$$

TO

$$
\begin{aligned}
& \Psi(X, Y)_{x}=-\frac{1}{\xi(a)}\left(\left|\begin{array}{cc}
\xi(a) & \xi(c) \\
\eta(a) & \eta(c)
\end{array}\right|+\left|\begin{array}{ll}
\xi(a) & \xi(b) \\
\zeta(a) & \zeta(b)
\end{array}\right|\right) \\
& \Omega(X, Y)_{x}=\frac{1}{\xi(a)}\left(\left|\begin{array}{cc}
\xi(a) & \xi(c) \\
\eta(a) & \eta(c)
\end{array}\right|-\left|\begin{array}{ll}
\xi(a) & \xi(b) \\
\zeta(a) & \zeta(b)
\end{array}\right|\right)
\end{aligned}
$$

В частности, если $a=e_{0}$ и $\xi=e^{0}$, т.е. $x=p\left(e_{0}\right) \times p\left(e^{0}\right)$, то, производя вычисления, найдем

$$
\Psi(X, Y)_{x}=-\eta_{k} c^{k}-\zeta_{k} b^{k}, \quad \Omega(X, Y)_{x}=\eta_{k} c^{k}-\zeta_{k} b^{k}
$$

Следовательно, $\Psi$ - псевдориманова метрика сигнатуры $(n, n)$, а $\Omega$ - почти симплектическая форма на $\mathfrak{N}$. Отсюда следует, в частности, что $\mathfrak{N}$ ориентируемо.

Заметим, что формулы (45) и (46) можно переписать в виде

$$
\begin{aligned}
& \Psi(X, Y)_{x}=-\frac{1}{\xi(a)}(\xi \wedge \eta(a, c)+\xi \wedge \zeta(a, b)) \\
& \Omega(X, Y)_{x}=\frac{1}{\xi(a)}(\xi \wedge \eta(a, c)-\xi \wedge \zeta(a, b)) .
\end{aligned}
$$

Формы $\Psi$ и $\Omega$ можно определить геометрически. Пусть $x=(A, \Xi) \in \mathfrak{N}$ - произвольная невырожденная нуль-пара, $A=\langle a\rangle$ и $\Xi=\langle\xi\rangle$. Тогда

$$
T_{x} \mathfrak{N}=T_{A} P_{n} \oplus T_{\Xi} P_{n}^{*} \equiv(W /\langle a\rangle) \oplus\left(W^{*} /\langle\xi\rangle\right)
$$


Для любой пары касательных векторов $X$ и $Y$ из $T_{x} \mathfrak{N}$ имеем

$$
X=X_{1} \oplus X^{1} \quad \text { и } \quad Y=Y_{1} \oplus Y^{1}
$$

где

$$
\begin{array}{ll}
X_{1}=\langle a\rangle+\bar{X}_{1} \in W /\langle a\rangle, & X^{1}=\langle\xi\rangle+\bar{X}^{1} \in W^{*} /\langle\xi\rangle, \\
Y_{1}=\langle a\rangle+\bar{Y}_{1} \in W /\langle a\rangle, & Y^{1}=\langle\xi\rangle+\bar{Y}^{1} \in W^{*} /\langle\xi\rangle .
\end{array}
$$

Если потребовать, чтобы выполнялись условия

$$
\xi\left(\bar{X}_{1}\right)=\bar{X}^{1}(a)=\xi\left(\bar{Y}_{1}\right)=\bar{Y}^{1}(a)=0,
$$

то для каждой пары касательных векторов $X$ и $Y$ из $T_{x} \mathfrak{N}$ будет однозначно определена пара векторов $\bar{X}_{1}$ и $\bar{Y}_{1}$ из $W$ и пара ковекторов $\bar{X}^{1}$ и $\bar{Y}^{1}$ из $W^{*}$. Теперь формы $\Psi$ и $\Omega$ можно определить так:

$$
\Psi_{x}(X, Y)=-\bar{X}^{1}\left(\bar{Y}_{1}\right)-\bar{Y}^{1}\left(\bar{X}_{1}\right), \quad \Omega_{x}(X, Y)=\bar{X}^{1}\left(\bar{Y}_{1}\right)-\bar{Y}^{1}\left(\bar{X}_{1}\right) .
$$

На многообразии $\mathfrak{N}$, несущем структуру почти произведения, внутренним образом определен эндоморфизм $\mathbf{J}: \mathfrak{X}(\mathfrak{N}) \rightarrow \mathfrak{X}(\mathfrak{N})$, действуюший по формуле

$$
\mathbf{J}_{x}\left(X_{1} \oplus X^{1}\right)=-X_{1} \oplus X^{1}
$$

или, подробнее,

$$
\mathbf{J}_{x}((\langle a\rangle+b) \oplus(\langle\xi\rangle+\eta))=(\langle a\rangle-b) \oplus(\langle\xi\rangle+\eta),
$$

где

$$
x=(\langle a\rangle,\langle\xi\rangle) \in \mathfrak{N}, \quad X_{1} \oplus X^{1}=\left(p_{a}\right)_{*} b \oplus\left(p_{\xi}\right)_{*} \eta=(\langle a\rangle+b) \oplus(\langle\xi\rangle+\eta) \in T_{x} \mathfrak{N} .
$$

Производя очевидные вычисления, получаем

$$
\mathbf{J}^{2}=\mathrm{id}, \quad \Psi(\mathbf{J} X, \mathbf{J} Y)=-\Psi(X, Y), \quad \Omega(X, Y)=\Psi(X, \mathbf{J} Y), \quad X, Y \in \mathfrak{X}(\mathfrak{N}) .
$$

Таким образом, пара $\{\Psi, \mathbf{J}\}$ является внутренним образом определенной почти эрмитовой структурой гиперболического типа на многообразии $\mathfrak{N}$. При этом почти симплектическая форма $\Omega$ является фундаментальной 2-формой этой структуры. Итак, доказано

ПРЕДЛОЖЕНИЕ 5. Многообразие невырожденных нуль-пар $\mathfrak{N}$ является $2 n$ мерным, связным, некомпактным, ориентируемым, вещественно-аналитическим, псевдоримановым и почти симплектическим многообразием, несущим естественную структуру прямого произведения и каноническую почти эрмитову структуру гиперболического типа. 


\section{2. Структура однородного пространства на многообразии невы-} рожденных нуль-пар. Пусть $\mathrm{GL}(n+1, \mathbb{R})$ - полная линейная группа порядка $n+1$. Зафиксируем базис $\left\{\varepsilon_{u}\right\}$ в $W$, и пусть $\left\{\varepsilon^{u}\right\}-$ сопряженный кобазис.

Левое действие $L: \operatorname{GL}(n+1, \mathbb{R}) \times \mathfrak{N} \rightarrow \mathfrak{N}$ группы $\mathrm{GL}(n+1, \mathbb{R})$ на многообразии невырожденных нуль-пар $\mathfrak{N}$ определим соотношением

$$
L:(g,(\langle a\rangle,\langle\xi\rangle)) \mapsto\left(\langle g a\rangle,\left\langle\xi g^{-1}\right\rangle\right) .
$$

Здесь $a \in W$ рассматривается как вектор-столбец, а $\xi \in W^{*}$ - как вектор-строка. Если нуль-пара $x=(\langle a\rangle,\langle\xi\rangle)$ невырождена, т.е. $\xi(a)=\xi \cdot a \neq 0$, то нуль-пара $L_{g} x=\left(\langle g a\rangle,\left\langle\xi g^{-1}\right\rangle\right)$, полученная левым сдвигом на $g \in \mathrm{GL}(n+1, \mathbb{R})$, также является невырожденной: $\left(\xi g^{-1}\right)(g a)=\xi(a) \neq 0$. Поэтому для любого $g \in \mathrm{GL}(n, \mathbb{R})$ отображение $L_{g}$ является автоморфизмом многообразия $\mathfrak{N}$. Так как $L_{h} \circ L_{g}=L_{h g}$, то отображение $L$ действительно является левым действием.

Докажем, что действие $L$ транзитивно. Пусть $x=(\langle a\rangle,\langle\xi\rangle)$ и $y=(\langle b\rangle,\langle\eta\rangle)-$ две невырожденные нуль-пары. Рассмотрим некоторьй базис $\left\{a_{1}, a_{2}, \ldots, a_{n}\right\}$ подпространства $W_{\xi}=\{X \in W \mid \xi(X)=0\}$, и пусть $\left\{b_{1}, b_{2}, \ldots, b_{n}\right\}$ - произвольньй базис в подпространстве $W_{\eta}=\{X \in W \mid \eta(X)=0\}$. Так как нуль-пары $x$ и $y$ невырождены, то $a \notin W_{\xi}$ и $b \notin W_{\eta}$. Следовательно, $A=\left\{a_{0}=a, a_{1}, a_{2}, \ldots, a_{n}\right\}$ и $B=\left\{b_{0}=b, b_{1}, b_{2}, \ldots, b_{n}\right\}$ - пара базисов пространства $W$. Базисы $A$ и $B$ отождествим с невырожденными матрицами $\left(A_{u}^{v}\right)$ и $\left(B_{u}^{v}\right)$, которые однозначно определяются из разложений $a_{u}=A_{u}^{v} \varepsilon_{v}$ и $b_{u}=B_{u}^{v} \varepsilon_{v}$. Рассмотрим невырожденную матрицу $g=\left(g_{u}^{v}\right)=\left(B_{w}^{v} \bar{A}_{u}^{w}\right)=B A^{-1} \in \mathrm{GL}(n+1, \mathbb{R})$ и докажем, что левый сдвиг на элемент $g$ переведет нуль-пару $x$ в нуль-пару $y$. Действительно, имеем $g=B A^{-1}, g^{-1}=A B^{-1}$. Следовательно,

$$
g a=B A^{-1} a=B_{u}^{v} \bar{A}_{w}^{u} A_{0}^{w} \varepsilon_{v}=B_{u}^{v} \delta_{0}^{u} \varepsilon_{v}=B_{0}^{v} \varepsilon_{v}=b .
$$

Кроме того, можем записать

$$
\begin{array}{ll}
\xi\left(a_{i}\right)=\xi \cdot a_{i}=\xi_{u} A_{i}^{u}=0, & \xi(a)=\xi_{u} A_{0}^{u} \neq 0, \\
\eta\left(b_{i}\right)=\eta \cdot b_{i}=\eta_{u} B_{i}^{u}=0, & \eta(b)=\eta_{u} B_{0}^{u} \neq 0 .
\end{array}
$$

Пусть $\left\{b^{u}\right\}$ - кобазис, сопряженный базису $\left\{b_{u}\right\}$. Тогда

$$
b^{u}=\bar{B}_{v}^{u} \varepsilon^{v}, \quad \varepsilon^{w}=B_{p}^{w} b^{p}, \quad \eta=\eta_{u} \varepsilon^{u}=\eta_{u} B_{v}^{u} b^{v}=\eta_{u} B_{0}^{u} b^{0}=\eta(b) b^{0} .
$$

Следовательно,

$$
\xi g^{-1}=\xi A B^{-1}=\xi_{u} A_{v}^{u} \bar{B}_{w}^{v} \varepsilon^{w}=\xi_{u} A_{0}^{u} \bar{B}_{w}^{0} B_{p}^{w} b^{p}=\xi(a) \delta_{p}^{0} b^{p}=\xi(a) b^{0}=\frac{\xi(a)}{\eta(b)} \eta .
$$

Итак,

$$
L_{g} x=L_{g}(\langle a\rangle,\langle\xi\rangle)=\left(\langle g a\rangle,\left\langle\xi g^{-1}\right\rangle\right)=\left(\langle b\rangle,\left\langle\frac{\xi(a)}{\eta(b)} \eta\right\rangle\right)=(\langle b\rangle,\langle\eta\rangle)=y .
$$


Таким образом, группа Ли $\mathrm{GL}(n+1, \mathbb{R})$ действует на $\mathfrak{N}$ транзитивно, т. е. $\mathfrak{N}$ - однородное пространство, причем $\mathfrak{N}=\mathrm{GL}(n, \mathbb{R}) / Z_{x}$, где $Z_{x}$ - подгруппа изотропии в некоторой фиксированной точке $x \in \mathfrak{N}$. Вычислим подгруппу изотропии в точке $x_{0}=\left(\left\langle\varepsilon_{0}\right\rangle,\left\langle\varepsilon^{0}\right\rangle\right)$ :

$$
g \in Z_{x_{0}} \Longleftrightarrow L_{g} x_{0}=x_{0} \Longleftrightarrow\left\{\begin{array}{l}
g \varepsilon_{0}=\lambda \varepsilon_{0} \\
\varepsilon^{0} g^{-1}=\mu \varepsilon^{0} \\
\lambda, \mu \neq 0
\end{array}\right\} \Longleftrightarrow g=\left(\begin{array}{cc}
g_{0}^{0} & 0 \\
0 & \left(g_{i}^{j}\right)
\end{array}\right) .
$$

Следовательно,

$$
Z_{x_{0}}=\left\{\left(\begin{array}{cc}
g_{0}^{0} & 0 \\
0 & \left(g_{i}^{j}\right)
\end{array}\right) \mid g_{0}^{0} \in \mathrm{GL}(1, \mathbb{R}),\left(g_{i}^{j}\right) \in \mathrm{GL}(n, \mathbb{R})\right\}
$$

т. е. $Z_{x_{0}} \cong \mathrm{GL}(1, \mathbb{R}) \times \mathrm{GL}(n, \mathbb{R})$.

Итак, многообразие невырожденных нуль-пар $\mathfrak{N}$ несет структуру однородного пространства:

$$
\mathfrak{N} \equiv \mathrm{GL}(n+1, \mathbb{R}) / \mathrm{GL}(1, \mathbb{R}) \times \mathrm{GL}(n, \mathbb{R}) .
$$

Пусть $\mathscr{F}(W)$ - совокупность всех базисов пространства $W$. Зафиксируем базис $\left\{\varepsilon_{u}\right\} \in \mathscr{F}(W)$. Тогда для любого базиса $\left\{e_{u}\right\} \in \mathscr{F}(W)$ существует единственная матрица $\left(C_{u}^{v}\right) \in \mathrm{GL}(n+1, \mathbb{R})$, определяемая условием $e_{u}=C_{u}^{v} \varepsilon_{v}$. Биективное соответствие между $\mathrm{GL}(n+1, \mathbb{R})$ и $\mathscr{F}(W)$ позволяет внести во множество базисов $\mathscr{F}(W)$ структуру аналитической групшы Ли $\mathrm{GL}(n+1, \mathbb{R})$. При этом выбор начального базиса $\left\{\varepsilon_{u}\right\}$ можно рассматривать как фиксацию глобальной карты на $\mathscr{F}(W)$, относительно которой компоненты матрищы $\left(C_{u}^{v}\right)$ есть координаты базиca $\left\{e_{u}\right\} \in \mathscr{F}(W)$. Итак, $\mathscr{F}(W) \equiv \mathrm{GL}(n+1, \mathbb{R})$.

Аналогично, фиксация базиса $\left\{\varepsilon^{u}\right\} \in \mathscr{F}\left(W^{*}\right)$ также позволяет отождествить многообразие всех базисов $\mathscr{F}\left(W^{*}\right)$ пространства $W^{*}$ с аналитической группой Ли $\mathrm{GL}(n+1, \mathbb{R})$. Таким образом, $\mathscr{F}\left(W^{*}\right) \equiv \mathrm{GL}(n+1, \mathbb{R})$.

Будем считать, что начальные базисы $\left\{\varepsilon_{u}\right\}$ и $\left\{\varepsilon^{u}\right\}$ являются сопряженными, т. е. $\varepsilon^{u}\left(\varepsilon_{v}\right)=\delta_{v}^{u}$. Тогда отображение $h: \mathscr{F}(W) \rightarrow \mathscr{F}\left(W^{*}\right)$, которое базису $\left\{e_{u}\right\}$ сопоставляет сопряженньй кобазис $\left\{e^{u}\right\}$, является диффеоморфизмом. Этот диффеоморфизм можно рассматривать как автоморфизм $h: \mathrm{GL}(n+1, \mathbb{R}) \rightarrow \mathrm{GL}(n+1$, $\mathbb{R})$ групшы Ли $\mathrm{GL}(n+1, \mathbb{R})$, при котором $h(g)=g^{-1}$. Ясно, что $h$ - аналитический диффеоморфизм.

На многообразиях $\mathscr{F}(W)$ и $\mathscr{F}\left(W^{*}\right)$ правое действие групшы $\mathrm{GL}(n+1, \mathbb{R})$ определяется так:

$$
\begin{gathered}
\left(\left\{e_{u}\right\},\left(G_{u}^{v}\right)\right) \mapsto\left\{\bar{e}_{u}\right\}, \quad \bar{e}_{u}=G_{u}^{v} e_{v}, \\
\left(\left\{e^{u}\right\},\left(G_{u}^{v}\right)\right) \mapsto\left\{\bar{e}^{u}\right\}, \quad \bar{e}^{u}=\bar{G}_{v}^{u} e^{v}, \quad\left(\bar{G}_{u}^{v}\right)=\left(G_{u}^{v}\right)^{-1} .
\end{gathered}
$$

Пусть $H$ - подгруппа в $\mathrm{GL}(n+1, \mathbb{R})$, определяемая следуюшим образом:

$$
H=\left\{\left(\begin{array}{cc}
G_{0}^{0} & 0 \\
0 & \left(G_{i}^{j}\right)
\end{array}\right) \in \mathrm{GL}(n+1, \mathbb{R}) \mid\left(G_{i}^{j}\right) \in \mathrm{GL}(n, \mathbb{R}), \quad G_{0}^{0} \neq 0\right\} .
$$


Ясно, что $H \cong \mathrm{GL}(1, \mathbb{R}) \times \mathrm{GL}(n, \mathbb{R})$.

Рассмотрим аналитическое отображение

$$
\pi=\left(p \circ e_{0}\right) \times\left(p \circ e^{0} \circ h\right): \mathscr{F}(W) \rightarrow \mathfrak{N}=\left(P(W) \times P\left(W^{*}\right)\right) \backslash M_{0},
$$

которое каждому базису $\left\{e_{u}\right\} \in \mathscr{F}(W)$ сопоставляет невырожденную нуль-пару $(A, \Xi) \in \mathfrak{N}$, порожденную вектором $e_{0}$ и ковектором $e^{0}$, т. е.

$$
\begin{gathered}
A=\left(p \circ e_{0}\right)\left(\left\{e_{u}\right\}\right)=p\left(e_{0}\right)=\left\langle e_{0}\right\rangle, \\
\Xi=\left(p \circ e^{0} \circ h\right)\left(\left\{e_{u}\right\}\right)=\left(p \circ e^{0}\right)\left(\left\{e^{u}\right\}\right)=p\left(e^{0}\right)=\left\langle e^{0}\right\rangle .
\end{gathered}
$$

Итак, $\pi\left(\left\{e_{u}\right\}\right)=(A, \Xi)=\left(\left\langle e_{0}\right\rangle,\left\langle e^{0}\right\rangle\right) \in \mathfrak{N}$.

Легко видеть, что группа $H$ совпадает с подгруппой изотропии нуль-пары $\pi\left(\left\{e_{u}\right\}\right)=\left(\left\langle e_{0}\right\rangle,\left\langle e^{0}\right\rangle\right)$. Следовательно, в силу естественных отождествлений $\mathscr{F}(W) \equiv \mathrm{GL}(n+1, \mathbb{R})$ и $H \cong \mathrm{GL}(1, \mathbb{R}) \times \mathrm{GL}(n, \mathbb{R})$ многообразие невырожденных нуль-пар $\mathfrak{N}$ можно рассматривать как факторпространство $\mathscr{F}(W) / H$, порожденное правым действием группы $H$ на многообразии $\mathscr{F}(W)$. С этой точки зрения нуль-пару $x=(A, \Xi)$ можно отождествить с совокупностью базисов $\left\{e_{u}\right\}$ из $\mathscr{F}(W)$, для каждого из которых $p\left(e_{0}\right)=A$ и $p\left(e^{0}\right)=\Xi$.

Таким образом, четверка $\mathbf{P}(\mathfrak{N})=(\mathscr{F}(W), \mathfrak{N}, H, \pi)$ - главное расслоение $\mathrm{c}$ тотальным пространством $\mathscr{F}(W) \equiv \mathrm{GL}(n+1, \mathbb{R})$, структурной группой $H \cong$ $\mathrm{GL}(1, \mathbb{R}) \times \mathrm{GL}(n, \mathbb{R})$, базой $\mathfrak{N} \equiv \mathrm{GL}(n+1, \mathbb{R}) / \mathrm{GL}(1, \mathbb{R}) \times \mathrm{GL}(n, \mathbb{R})$ и проекцией $\pi=\left(p \circ e_{0}\right) \times\left(p \circ e^{0} \circ h\right)$.

Найдем структурные уравнения расслоения $\mathbf{P}(\mathfrak{N})$. Каждый вектор $e_{u}$ базиса $B=\left\{e_{v}\right\}$ можно рассматривать как гладкую $W$-значную функцию на $\mathscr{F}(W) \equiv$ $\operatorname{GL}(n+1, \mathbb{R}), e_{u}:\left\{e_{v}\right\} \mapsto e_{u}$. Тогда $d e_{u}-$ это $W$-значная 1 -форма на $\mathrm{GL}(n+1$, $\mathbb{R})$. Раскладывая форму $d e_{u}$ по базису $B$, получим так называемые уравнения инфинитезимальных преобразований многообразия реперов $\mathscr{F}(W)$ :

$$
d e_{u}=\omega_{u}^{v} \otimes e_{v} .
$$

Аналогично, для многообразия $\mathscr{F}\left(W^{*}\right)$ имеем $d e^{u}=\bar{\omega}_{v}^{u} \otimes e^{v}$. Дифференцируя уравнения $e^{u}\left(e_{v}\right)=\delta_{v}^{u}$, найдем, что $\bar{\omega}_{u}^{v}=-\omega_{u}^{v}$. Следовательно, уравнения инфинитезимальных преобразований многообразия реперов $\mathscr{F}\left(W^{*}\right)$ примут вид

$$
d e^{u}=-\omega_{v}^{u} \otimes e^{v} .
$$

Легко видеть, что 1-формы $\omega_{u}^{v}$, входяшие в (47) и (48), при надлежаших отождествлениях совпадают с формами Маурера-Картана группы Ли $\mathrm{GL}(n+1, \mathbb{R})$, удовлетворяюшими структурным уравнениям

$$
d \omega_{u}^{v}=\omega_{u}^{w} \wedge \omega_{w}^{v}
$$


Уравнения (49) получаются после применения оператора внешнего дифференцирования либо к системе (47), либо к системе (48).

Так как для любого $X \in \mathfrak{X}(\mathscr{F}(W))$ имеют место равенства

$$
\begin{aligned}
& \left(p \circ e_{0}\right)_{*}(X)_{B}=\left\langle e_{0}\right\rangle+\omega_{0}^{i}(X) e_{i} \in W /\left\langle e_{0}\right\rangle, \\
& \left(p \circ e^{0}\right)_{*}(X)_{B}=\left\langle e^{0}\right\rangle-\omega_{i}^{0}(X) e^{i} \in W^{*} /\left\langle e^{0}\right\rangle,
\end{aligned}
$$

TO

$$
(\pi)_{*}(X)_{B}=\left(\left\langle e_{0}\right\rangle+\omega_{0}^{i}(X) e_{i}\right) \oplus\left(\left\langle e^{0}\right\rangle-\omega_{i}^{0}(X) e^{i}\right) \in T_{\left\langle e_{0}\right\rangle \times\left\langle e^{0}\right\rangle} \mathfrak{N} .
$$

Следовательно, формы $\omega^{i}=\omega_{0}^{i}$ и $\omega_{i}=\omega_{i}^{0}$ образуют базис пространства горизонтальных 1-форм расслоения $\mathbf{P}(\mathfrak{N})$. Из (49) находим, что первая группа структурных уравнений расслоения $\mathbf{P}(\mathfrak{N})$ имеет вид

$$
d \omega^{i}=\omega^{j} \wedge \theta_{j}^{i}, \quad d \omega_{i}=\theta_{i}^{j} \wedge \omega_{j}
$$

Здесь

$$
\theta_{i}^{j}=\omega_{i}^{j}-\delta_{i}^{j} \omega_{0}^{0}
$$

Применяя оператор внешнего дифференщирования к тождествам (51) и используя (49), получим вторую группу структурных уравнений расслоения $\mathbf{P}(\mathfrak{N})$ :

$$
d \theta_{i}^{j}=\theta_{i}^{k} \wedge \theta_{k}^{j}+\omega_{i} \wedge \omega^{j}+\delta_{i}^{j} \omega_{k} \wedge \omega^{k}
$$

Кроме того, из уравнений (49) находим

$$
d \omega_{0}^{0}=\omega^{i} \wedge \omega_{i}
$$

Система уравнений (50)-(53) эквивалентна системе (49) и является системой уравнений Маурера-Картана группы Ли $\mathrm{GL}(n+1, \mathbb{R})$. Ограничение системы (52), (53) на слой расслоения $\mathbf{P}(\mathfrak{N})$ принимает вид

$$
d \theta_{i}^{j}=\theta_{i}^{k} \wedge \theta_{k}^{j}, \quad d \omega_{0}^{0}=0
$$

Легко видеть, что (54) - это система структурных уравнений Маурера-Картана группы Ли $H \cong \mathrm{GL}(1, \mathbb{R}) \times \mathrm{GL}(n, \mathbb{R})$.

Систему (50)-(53) назовем структурными уравнениями многообразия невырожсденньх нуль-пар $\mathfrak{N} \equiv \mathrm{GL}(n+1, \mathbb{R}) / \mathrm{GL}(1, \mathbb{R}) \times \mathrm{GL}(n, \mathbb{R})$.

В терминах горизонтальных 1-форм $\omega^{i}$ и $\omega_{j}$ псевдориманова метрика и 2-форорма $\Omega$, определенные в п. 2.1, могут быть представлены в виде

$$
\begin{aligned}
& \Psi(X, Y)=2 \omega^{i} \odot \omega_{i}(X, Y)=\omega^{i}(X) \omega_{i}(Y)+\omega^{i}(Y) \omega_{i}(X), \\
& \Omega(X, Y)=2 \omega^{i} \wedge \omega_{i}(X, Y)=\omega^{i}(X) \omega_{i}(Y)-\omega^{i}(Y) \omega_{i}(X),
\end{aligned}
$$


где $X, Y \in \mathfrak{X}(\mathfrak{N})$. Из последнего соотношения, а также из (53) находим, что $\Omega=$ $d \omega_{0}^{0}$. Следовательно, 2 -форма $\Omega$ является точной, в частности замкнутой, а значит, определяет симплектическую структуру на многообразии $\mathfrak{N}$. Итак, многообразие невырожденных нуль-пар является симплектическим многообразием.

Структура почти произведения на $\mathfrak{N}$ определяется парой $n$-мерных взаимно дополнительных инволютивных распределений, задаваемых системами Пфаффа $\omega^{i}=0$ и $\omega_{i}=0$. Интегральными многообразиями первой системы являются подмногообразия невырожденных нуль-пар $(A, \Xi)$ с фиксированной точкой $A$, а интегральными многообразиями второй системы-подмногообразия невырожденных нуль-пар с фиксированной гиперплоскостью $\Xi$. Каждое такое подмногообразие диффеоморфно $n$-мерному аффинному пространству.

2.3. Вложение $2 n$-мерного многообразия нуль-пар в проективное пространство размерности $n^{2}+2 n$. Пусть $W \otimes W^{*}$ - пространство тензоров типа $(1,1)$ над векторным пространством $W$. Рассматривая $W \otimes W^{*}$ как $(n+1)^{2}$-мерное линейное пространство, обозначим через $P_{K}=P\left(W \otimes W^{*}\right)$ проективное пространство размерности $K=n^{2}+2 n$, порожденное пространством $W \otimes W^{*}$. Пусть $\left\{\varepsilon_{u}^{v}=\varepsilon_{u} \otimes \varepsilon^{v}\right\}-$ стандартный базис в $W \otimes W^{*}$, порожденный фиксированной парой сопряженных базисов $\left\{\varepsilon_{u}\right\} \in \mathscr{F}(W)$ и $\left\{\varepsilon^{v}\right\} \in \mathscr{F}\left(W^{*}\right)$. Если $t=X_{u}^{v} \varepsilon_{v}^{u} \in W \otimes W^{*}-$ произвольный ненулевой тензор, то упорядоченный набор чисел $\left(X_{u}^{v}\right)$ является однородными координатами точки $T=p(t) \in P_{K}$.

Рассмотрим вложение Сегре

$$
f_{s}: P(W) \times P\left(W^{*}\right) \rightarrow P\left(W \otimes W^{*}\right)
$$

многообразия нуль-пар $M=P_{n} \times P_{n}^{*}=P(W) \times P\left(W^{*}\right)$ в проективное пространство $P_{K}=P\left(W \otimes W^{*}\right)$. Это отображение задается следующим образом:

$$
f_{s}: p(a) \times p(\xi) \mapsto p(a \otimes \xi) .
$$

$\mathrm{B}$ координатной форме это вложение определяется уравнениями $\rho X_{u}^{v}=a^{v} \xi_{u}$.

Ясно, что $f_{s}(M)$ - аналитическоеподмногообразие в $P_{K}$. Многообразие вырожденных нуль-пар $f_{s}\left(M_{0}\right)$ есть пересечение $f_{s}(M)$ с гиперплоскостью

$$
X_{0}^{0}+X_{1}^{1}+\cdots+X_{n}^{n}=0
$$

пространства $P_{K}$.

Многообразие невырожденных нуль-пар $f_{s}(\mathfrak{N})$, вложенное в $P_{K}$, будем обозначать через $\mathfrak{W}$. Пусть $\mathscr{F}\left(W \otimes W^{*}\right)$ - многообразие всех базисов пространства $W \otimes W^{*}$. Ясно, что

$$
\mathscr{F}\left(W \otimes W^{*}\right) \equiv \operatorname{GL}(K, \mathbb{R})=\left\{\left(G_{u v}^{w p}\right) \mid \operatorname{det}\left(G_{u v}^{w p}\right) \neq 0\right\} .
$$

Здесь и в дальнейшем строки и столбцы квадратных матриц порядка $K=(n+1)^{2}$ нумеруются парами индексов. Правое действие групшы $\mathrm{GL}(K, \mathbb{R})$ на многообразии $\mathscr{F}\left(W \otimes W^{*}\right)$ определяется следуюшим образом:

$$
\left(\left\{e_{u}^{v}\right\},\left(G_{u w}^{v p}\right)\right) \mapsto\left\{\bar{e}_{u}^{v}\right\}, \quad \bar{e}_{u}^{v}=G_{u w}^{v p} e_{p}^{w} .
$$


Вложение $f_{s}$ индуцирует отображение $\hat{f}_{s}: \mathscr{F}(W) \rightarrow \mathscr{F}\left(W \otimes W^{*}\right)$ по следуюшему закону:

$$
\hat{f}_{s}:\left\{e_{u}\right\} \mapsto\left\{e_{u} \otimes e^{v}\right\}, \quad\left\{e^{v}\right\}=h\left(\left\{e_{u}\right\}\right) .
$$

В координатах, соответствующих естественным отождествлениям $\mathscr{F}(W) \equiv$ $\mathrm{GL}(n+1, \mathbb{R})$ и $\mathscr{F}\left(W \otimes W^{*}\right) \equiv \mathrm{GL}(K, \mathbb{R})$, отображение $\hat{f}_{s}$ определяется уравнениями

$$
C_{u w}^{v p}=C_{u}^{p} \bar{C}_{w}^{v},
$$

где $\left(C_{u}^{p}\right) \in \mathrm{GL}(n+1, \mathbb{R}),\left(\bar{C}_{w}^{v}\right)=\left(C_{u}^{p}\right)^{-1},\left(C_{u w}^{v p}\right) \in \mathrm{GL}(K, \mathbb{R})$. Ясно, что $\hat{f}_{s}-$ аналитическое вложение. Обозначим через $\mathscr{F}(\mathfrak{W})=\operatorname{Im} \hat{f}_{s}$ образ многообразия $\mathscr{F}(W)$ при вложении $\hat{f}_{s}$.

Гомоморфизм $\sigma$ подгруппы изотропии $H \cong \mathrm{GL}(1, \mathbb{R}) \times \mathrm{GL}(n, \mathbb{R})$ нуль-пары $\left(\left\langle e_{0}\right\rangle,\left\langle e^{0}\right\rangle\right)$ в группу $\mathrm{GL}(K, \mathbb{R})$ определяется по следуюшему закону:

$$
\sigma: \mathrm{GL}(1, \mathbb{R}) \times \mathrm{GL}(n, \mathbb{R}) \ni\left(\begin{array}{cc}
g & 0 \\
0 & \left(g_{i}^{j}\right)
\end{array}\right) \mapsto\left(\begin{array}{cccc}
g & 0 & 0 & 0 \\
0 & \bar{g}\left(g_{i}^{j}\right) & 0 & 0 \\
0 & 0 & g\left(\bar{g}_{i}^{j}\right) & 0 \\
0 & 0 & 0 & \left(g_{i}^{j} \bar{g}_{k}^{l}\right)
\end{array}\right) .
$$

Здесь $\left(\bar{g}_{i}^{j}\right)=\left(g_{i}^{j}\right)^{-1} \in \mathrm{GL}(n, \mathbb{R}), \bar{g}=\frac{1}{g} \in \mathrm{GL}(1, \mathbb{R})$. Действие групшы $H$ на многообразии $\mathscr{F}(\mathfrak{W})$ определяется по формулам $\left(\left\{e_{u}^{v}\right\},\left\{g,\left(g_{i}^{j}\right)\right\}\right) \mapsto\left\{\bar{e}_{u}^{v}\right\}$, где $\bar{e}_{0}^{0}=$ $g e_{0}^{0}, \bar{e}_{i}^{0}=\bar{g} g_{i}^{j} e_{j}^{0}, \bar{e}_{0}^{i}=g \bar{g}_{j}^{i} e_{0}^{j}, \bar{e}_{i}^{j}=g_{i}^{k} \bar{g}_{l}^{j} e_{k}^{l}$.

Проекция $\pi_{0}^{0}=p \circ e_{0}^{0}: \mathscr{F}(\mathfrak{W}) \rightarrow \mathfrak{W}$ является аналитическим отображением. Четверка $\mathbf{P}(\mathfrak{W})=\left(\mathscr{F}(\mathfrak{W}), \mathfrak{W}, H, \pi_{0}^{0}\right)$ есть главное расслоение, изоморфное расслоению $\mathbf{P}(\mathfrak{N})=(\mathscr{F}(W), \mathfrak{N}, H, \pi)$. Изоморфизм определяется посредством отображений $f_{s}, \hat{f}_{s}$ и $\sigma$, где $f_{s}: \mathfrak{N} \rightarrow \mathfrak{W} \subset P\left(W \otimes W^{*}\right)$ - аналитический диффеоморфизм на образ, $\hat{f}_{s}: \mathscr{F}(W) \rightarrow \mathscr{F}(\mathfrak{W}) \subset \mathscr{F}\left(W \otimes W^{*}\right)$ - аналитический диффеоморфизм на образ, $\sigma: H \rightarrow \mathrm{GL}(K, \mathbb{R})$ - аналитический изоморфизм на образ, которые, как легко видеть, обладают следующими свойствами:

$$
\begin{aligned}
\pi \circ f_{s}=\hat{f}_{s} \circ \pi_{0}^{0}, \\
\sigma\left(g_{1} \cdot g_{2}\right)=\sigma\left(g_{1}\right) \cdot g_{2}, \quad g_{1}, g_{2} \in H .
\end{aligned}
$$

Уравнения инфинитезимальных преобразований для многообразия реперов $\mathscr{F}\left(W \otimes W^{*}\right)$ записываются в виде

$$
d e_{u}^{v}=\omega_{u w}^{v p} \otimes e_{p}^{w},
$$

где формы $\omega_{u w}^{v p}$ удовлетворяют структурным уравнениям Маурера-Картанагруппы Ли $\mathrm{GL}(K, \mathbb{R})$ :

$$
d \omega_{u w}^{v p}=\omega_{u r}^{v q} \wedge \omega_{q w}^{r p} .
$$

Дифференцируя уравнения $e_{u}^{v}=e_{u} \otimes e^{v}$, найдем ограничение 1 -форм $\omega_{u w}^{v p}$ на подмногообразие $\mathscr{F}(\mathfrak{W})$ :

$$
\hat{f}_{s}^{*} \omega_{u w}^{v p}=\omega_{u}^{v} \delta_{w}^{p}-\delta_{u}^{v} \omega_{w}^{p} .
$$


Следовательно, на $\mathscr{F}(\mathfrak{W})$ имеем

$$
\begin{gathered}
d e_{0}^{0}=\omega^{i} \otimes e_{i}^{0}-\omega_{i} \otimes e_{0}^{i}, \\
d e_{i}^{0}=\theta_{i}^{j} \otimes e_{j}^{0}-\omega_{j} \otimes \varepsilon_{i}^{j}, \quad d e_{0}^{i}=\omega^{j} \otimes \varepsilon_{j}^{i}-\theta_{j}^{i} \otimes e_{0}^{j}, \\
d \varepsilon_{i}^{j}=\left(\theta_{i}^{k} \delta_{l}^{j}-\delta_{i}^{k} \theta_{l}^{j}\right) \otimes \varepsilon_{k}^{l}+\left(\omega_{i} \delta_{k}^{j}+\delta_{i}^{j} \omega_{k}\right) \otimes e_{0}^{k}-\left(\omega^{j} \delta_{i}^{k}+\delta_{i}^{j} \omega^{k}\right) \otimes e_{k}^{0},
\end{gathered}
$$

где $\varepsilon_{i}^{j}=e_{i}^{j}-\delta_{i}^{j} e_{0}^{0}$. Из последних соотношений находим, что

$$
P T_{x}(\mathfrak{W})=\left\langle e_{0}^{0}, e_{i}^{0}, e_{0}^{j}\right\rangle
$$

- проективная касательная плоскость к подмногообразию $\mathfrak{W}$ в точке $x=p\left(e_{0}^{0}\right)$;

$$
P T_{x}^{2}(\mathfrak{W})=P\left(W \otimes W^{*}\right)=P_{K}
$$

- соприкасающееся пространство к подмногообразию $\mathfrak{W}$ в точке $x$;

$$
\Phi_{x}(X, Y)=\left\langle e_{0}^{0}, e_{i}^{0}, e_{0}^{j}\right\rangle-\left(\omega^{i}(X) \omega_{j}(Y)+\omega^{i}(Y) \omega_{j}(X)\right) \varepsilon_{i}^{j}
$$

- вторая фундаментальная форма вложения $f_{s}$, рассматриваемая как отображение

$$
\Phi_{x}: T_{x}(\mathfrak{W}) \times T_{x}(\mathfrak{W}) \rightarrow W \otimes W^{*} /\left\langle e_{0}^{0}, e_{i}^{0}, e_{0}^{j}\right\rangle .
$$

2.4. Каноническая $\mathscr{K}_{1}$-структура на многообразии невырожденных нуль-пар. Введем обозначения:

$$
\begin{gathered}
I, J, \ldots=1,2, \ldots, 2 n ; \\
i, j, \ldots=1,2, \ldots, n ; \quad \hat{i}, \hat{j}, \ldots=n+1, n+2, \ldots, 2 n ; \\
\omega^{\hat{i}}=\omega_{i} ; \quad \theta_{\hat{i}}^{\hat{j}}=-\theta_{j}^{i} .
\end{gathered}
$$

Тогда уравнения (50), (52) можно выписать в виде

$$
\begin{gathered}
d \omega^{i}=\omega^{j} \wedge \theta_{j}^{i}, \quad d \omega^{\hat{i}}=\omega^{\hat{j}} \wedge \theta_{\hat{j}}^{\hat{i}}, \\
d \theta_{j}^{i}-\theta_{j}^{k} \wedge \theta_{k}^{i}=\left(\delta_{k j} \delta_{l}^{i}+\delta_{j}^{i} \delta_{k l}\right) \omega^{\hat{k}} \wedge \omega^{l}, \\
d \theta_{\hat{j}}^{\hat{i}}-\theta_{\hat{j}}^{\hat{k}} \wedge \theta_{\hat{k}}^{\hat{i}}=\left(\delta_{k j} \delta_{l}^{i}+\delta_{j}^{i} \delta_{k l}\right) \omega^{k} \wedge \omega^{\hat{l}} .
\end{gathered}
$$

Компоненты псевдоримановой метрики $\Psi=\omega^{i} \odot \omega_{i}=g_{I J} \omega^{I} \odot \omega^{J}$ имеют вид

$$
\left(g_{I J}\right)=\left(\begin{array}{cc}
0 & I_{n} \\
I_{n} & 0
\end{array}\right)
$$


где $I_{n}$ - единичная матрица порядка $n$. Легко проверить, что на $\mathfrak{N}$ выполняется система дифференциальных уравнений

$$
\nabla g_{I J} \equiv d g_{I J}-g_{L J} \theta_{I}^{L}-g_{I L} \theta_{J}^{L}=0
$$

Следовательно, с точностью до знака, $\Theta=\left(\begin{array}{cc}\left(\theta_{i}^{j}\right) & 0 \\ 0 & -\left(\theta_{i}^{j}\right)^{\top}\end{array}\right)$ - форма римановой связности $\nabla$ псевдоримановой метрики $\Psi$ на $\mathfrak{N}$, a $\omega=\left\{\omega^{I}\right\}=\left\{\omega^{i}, \omega^{\hat{i}}\right\}-$ форма смешения.

Фундаментальная форма

$$
\Omega=d \omega_{0}^{0}=\omega^{i} \wedge \omega_{i}=S_{I J} \omega^{I} \wedge \omega^{J},
$$

где $\left(S_{I J}\right)=\left(\begin{array}{cc}0 & I_{n} \\ -I_{n} & 0\end{array}\right)$, ковариантно-постоянна относительно связности $\nabla$ :

$$
\nabla S_{I J} \equiv d S_{I J}-S_{L J} \theta_{I}^{L}-S_{I L} \theta_{J}^{L}=0 .
$$

Из соотношений (56), (57) находим, что компоненты тензора Римана-Кристофеля связности $\nabla$ имеют вид

$$
R_{i \hat{j} k \hat{l}}=R_{\hat{i j k} \hat{k} l}=\delta_{k j} \delta_{i l}+\delta_{i j} \delta_{k l} \text {. }
$$

Остальные компоненты тензора кривизны либо равны нулю, либо находятся из известных свойств симметрии.

Как отмечалось ранее, на многообразии $\mathfrak{N}=\mathbb{R} P_{n} \diamond \mathbb{R} P_{n}$, несушем структуру почти произведения, внутренним образом определен эндоморфизм $\mathbf{J}: \mathfrak{X}(\mathfrak{N}) \rightarrow$ $\mathfrak{X}(\mathfrak{N})$ по формуле

$$
\Psi(X, \mathbf{J} Y)=\Omega(X, Y), \quad X, Y \in \mathfrak{X}(M) .
$$

Он обладает следуюшими свойствами:

$$
\mathbf{J}^{2}=\mathrm{id}, \quad \Psi(\mathbf{J} X, \mathbf{J} Y)=-\Psi(X, Y) .
$$

Из сказанного выше немедленно следует, что $\nabla \mathbf{J}=0$, т. е. пара $\{\Psi, \mathbf{J}\}$ является внутренним образом определенной келеровой структурой гиперболического muпа на многообразии $\mathfrak{N}$, причем в силу (58) многообразие $\mathfrak{N}$ является натуральной пространственной формой [5] относительно этой структуры. Более того, свертка тензора кривизны с метрическим тензором показывает, что компоненты тензора Риччи многообразия $\mathfrak{N}$ имеют вид

$$
\left(r_{I J}\right)=\left(\begin{array}{cc}
0 & (n+1) I_{n} \\
(n+1) I_{n} & 0
\end{array}\right) .
$$

Следовательно, $r_{I J}=(n+1) g_{I J}$, т.е. $\mathfrak{N}$ - многообразие Эйнштейна $c$ космологической константой $n+1$. Скалярная кривизна многообразия $\mathfrak{N}$ равна $r=2 n(n+1)$. Доказано 
ПРЕДЛОЖЕНИЕ 6. Пара $\{\Psi, \mathbf{J}\}$ является келеровой структурой зиперболического типа на многообразии $\mathfrak{N}$. При этом многообразие $\mathfrak{N}$ является натуральной пространственной формой относительно әтой структуры. Кроме того, $\mathfrak{N}$ - многообразие Эйнштейна.

Пусть $X, Y$ - пара касательных векторов, $X=X_{1}+X_{2}, Y=Y_{1}+Y_{2}-$ их каноническое разложение относительно структуры прямого произведения на $\mathfrak{N}$, а $(\cdot, \cdot)$ - стандартное скалярное произведение в $\mathbb{R}^{n}$. Тогда

$$
\Psi(X, Y)=\left(X_{1}, Y_{2}\right)+\left(Y_{1}, X_{2}\right), \quad \Omega(X, Y)=\left(X_{1}, Y_{2}\right)-\left(Y_{1}, X_{2}\right)
$$

а секционная кривизна в двумерном направлении $X \wedge Y$ вычисляется по формуле

$$
k(X \wedge Y)=2 \cdot \frac{(\Omega(X, Y))^{2}+\left|\begin{array}{cc}
\left(X_{1}, Y_{2}\right) & \left(Y_{1}, Y_{2}\right) \\
\left(X_{1}, X_{2}\right) & \left(Y_{1}, X_{2}\right)
\end{array}\right|}{(\Omega(X, Y))^{2}+4 \cdot\left|\begin{array}{ll}
\left(X_{1}, Y_{2}\right) & \left(Y_{1}, Y_{2}\right) \\
\left(X_{1}, X_{2}\right) & \left(Y_{1}, X_{2}\right)
\end{array}\right|} .
$$

Эту формулу можно переписать в виде

$$
k(X \wedge Y)=\frac{1}{2}+\frac{3}{2} \cdot \frac{(\Omega(X, Y))^{2}}{(\Psi(X, Y))^{2}-\Psi(X, X) \Psi(Y, Y)} .
$$

Из этой формулы снова вытекает, что многообразие $\mathfrak{N}$ является натуральной пространственной формой, а именно многообразием постоянной голоморфной секционной кривизны 2.

Разумеется, гомотетично преобразуя метрику $\Psi$ и форму $\Omega$, можно получить любое ненулевое значение ее голоморфной секционной кривизны при том же структурном операторе $\mathbf{J}$.

Из (59) вытекает

ПРЕДЛОЖЕНИЕ 7. Пусть $\mathscr{L}$ - вполне вещественное (или, что то жее самое, лагранжево) подмногообразие многообразия $\mathbb{R} P_{n} \diamond \mathbb{R} P_{n}$. Тогда в любом неизотропном двумерном направлении секиионная кривизна подмногообразия $\mathscr{L}$ постоянна и равна $\frac{1}{2}$.

(Сp. с теоремой 5.)

2.5. Геометрия лагранжевых подмногообразий многообразия $\mathbb{R} P_{n} \diamond$ $\mathbb{R} P_{n}$. Известно, что наибольшая размерность лагранжева подмногообразия $\mathscr{L}$ на $2 n$-мерном симплектическом многообразии равна $n$. Опишем лагранжевы подмногообразия максимальной размерности на многообразии невырожденных нуль-пар.

Сначала напомним, что подмногообразие $\mathscr{L}$ псевдориманова многообразия $\mathfrak{J}$ называется вполне изотропным, если касательные пространства подмногообразия $\mathscr{L}$ являются плоскими образуюшими изотропного конуса, определяющего псевдориманову метрику на $\mathfrak{W}$. Если метрический тензор на $\mathfrak{W}$ имеет сигнатуру $(n, n)$, 
то максимально возможная размерность вполне изотропного подмногообразия $\mathscr{L}$ (если оно существует) равна $n$.

Пусть $f: \mathscr{L} \rightarrow \mathfrak{W}$-вложение $n$-мерного многообразия в многообразие невырожденных нуль-пар $\mathfrak{W}$. Тогда ранг системы 1-форо

$$
\left\{f^{*} \omega^{1}, f^{*} \omega^{2}, \ldots, f^{*} \omega^{n}, f^{*} \omega_{1}, f^{*} \omega_{2}, \ldots, f^{*} \omega_{n}\right\}
$$

увлеченных с $\mathfrak{W}$ на $\mathscr{L}$, равен $n$. Подмногообразие $\mathscr{L}$ будет лагранжевым в том и только том случае, если $f^{*} \omega^{i} \wedge f^{*} \omega_{i} \equiv 0$. Подмногообразие $\mathscr{L}$ будет вполне изотропным тогда и только тогда, когда $f^{*} \omega^{i} \odot f^{*} \omega_{i} \equiv 0$. Наконец, подмногообразие $\mathscr{L}$ будет вполне изотропным и лагранжевым в том и только том случае, если $f^{*} \omega^{i} \otimes f^{*} \omega_{i} \equiv 0$. В дальнейшем, чтобы не усложнять обозначения, формы $f^{*} \omega^{i}$ и $f^{*} \omega_{i}$ будем снова обозначать через $\omega^{i}$ и $\omega_{i}$, подразумевая, что эти формы определены на $\mathscr{L}$ и их ранг равен $n$.

Опишем несколько конструктивных примеров лагранжевых подмногообразий.

ПримеР 1. Пусть $\Xi_{0} \in P_{n}^{*}$ - фиксированная гиперплоскость проективного пространства $P_{n}, A_{n}=P_{n} \backslash \Xi_{0}-$ множество всех точек пространства $P_{n}$, не лежаших в $\Xi_{0}$ (т.е. $A_{n}$ - афффинное пространство с "несобственной" гиперплоскостью $\left.\Xi_{0}\right)$. Обозначим через $\mathscr{L}$ совокупность всех нуль-пар $(A, \Xi)$ таких, что $A \in A_{n}$, $\Xi=\Xi_{0}$. Ясно, что $\mathscr{L}$ - гладкое $n$-мерное подмногообразие в $\mathfrak{N}$, диффеоморфное аффинному пространству $A_{n}$. При вложении в $P_{K}$ подмногообразие $\mathscr{L}$ задается системой параметрических уравнений $\rho X_{u}^{v}=a^{v} \xi_{u}^{0}, \xi_{u}^{0} a^{u} \neq 0$, где $\left\{\xi_{u}^{0}\right\}-$ тангенциальные координаты гиперплоскости $\Xi$. Ясно, что $f_{s}(\mathscr{L})$ - плоская образующая на $\mathfrak{W}$. Пусть $\mathscr{F}(\mathscr{L})$ - подмногообразие в $\mathscr{F}(W)$, определяемое условием $\Xi_{0}=p\left(e^{0}\right)=p\left(\left\langle e_{1}, e_{2}, \ldots, e_{n}\right\rangle\right)$. Дифференциальные уравнения подмногообразия $\mathscr{F}(\mathscr{L})$ имеют вид

$$
\omega_{i}=0
$$

Эта система вполне интегрируема, и, как отмечалось ранее, ее интегральными многообразиями на $\mathfrak{W}$ являются $n$-мерные плоские образующие. Кроме того, в силу этой системы имеет место тождество $\omega^{i} \otimes \omega_{i} \equiv 0$. Следовательно, подмногообразие $\mathscr{L}$ вполне изотропно и лагранжево. Очевидно также, что $\mathscr{L}$ вполне геодезично.

ПримеР 2. Рассмотрим конструкцию, двойственную примеру 1. Пусть $A_{0} \in$ $P_{n}$ - фиксированная точка проективного пространства $P_{n}, A_{n}^{*}$ - множество всех гиперплоскостей пространства $P_{n}$, не проходящих через $A_{0}$ (т.е. $A_{n}^{*}$ - аффинное пространство, двойственное $\left.A_{n}\right)$. Обозначим через $\mathscr{L}$ совокупность всех нуль-пар $(A, \Xi)$ таких, что $A=A_{0}, \Xi \in A_{n}^{*}$. Ясно, что $\mathscr{L}$ - гладкое $n$-мерное подмногообразие в $\mathfrak{N}$, диффеоморфное аффинному пространству $A_{n}$. При вложении в $P_{K}$ подмногообразие $\mathscr{L}$ задается системой параметрических уравнений $\rho X_{u}^{v}=a_{0}^{v} \xi_{u}$, $\xi_{u} a_{0}^{u} \neq 0$, где $\left\{a_{0}^{u}\right\}$ - однородные координаты точки $A_{0}$. Ясно, что $f_{s}(\mathscr{L})$ - плоская образуюшая на $\mathfrak{W}$. Пусть $\mathscr{F}(\mathscr{L})$ - подмногообразие в $\mathscr{F}(W)$, определяемое условием $A_{0}=p\left(e_{0}\right)$. Дифференциальные уравнения подмногообразия $\mathscr{F}(\mathscr{L})$ имеют вид

$$
\omega^{i}=0
$$


Эта система вполне интегрируема, и ее интегральными многообразиями на $\mathfrak{W}$ являются $n$-мерные плоские образующие. Ясно, что подмногообразие $\mathscr{L}$ вполне изотропно, лагранжево и вполне геодезично.

Таким образом, справедливо

УТВЕРЖДЕНИЕ 1. Пусть $\mathscr{L}$ - множсество нуль-пар из примера 1 или из примера 2. Тогда $\mathscr{L} \subset \mathfrak{N}$ - гладкое $n$-мерное подмногообразие, диффеоморфное аффинному пространству $A_{n}$. Это подмногообразие является лагранжевылм, вполне геодезическим и вполне изотропным. Образ $f_{s}(\mathscr{L})$ многообразия $\mathscr{L}$ при вложсении $f_{s}: \mathfrak{N} \rightarrow \mathfrak{W} \subset P_{K}$ есть плоская образующая на $\mathfrak{W}$. Через каждую точку многообразия ్ проходит единственное подмногообразие $\mathscr{L}$ из примера 1 и единственное подмногообразие $\mathscr{L}$ из примера 2.

Отметим, что интегрируемость на 以् систем Пфаффа (60) или (61) является очевидным следствием интегрируемости гладкой структуры почти произведения на $\mathfrak{N}$.

ПримеР 3 . Пусть $P_{r}$ - некоторая $r$-плоскость пространства $P_{n}$, в которой фиксирована "несобственная" гиперплоскость $P_{r-1} \subset P_{r}$. Множество $A_{r}=P_{r} \backslash$ $P_{r-1}$ есть $r$-мерное аффинное пространство, которое состоит из точек, принадлежащих $P_{r}$ и не принадлежащих $P_{r-1}$. Совокупность всех гиперплоскостей в $P_{n}$, проходящих через плоскость $P_{r-1}$, есть $(n-r)$-мерное проективное пространство $P_{n-r}^{*} \subset P_{n}^{*}$. Пусть $P_{n-r-1}^{*} \subset P_{n-r}^{*}-$ "несобственная" гиперплоскость в $P_{n-r}^{*}$, состоящая из тех гиперплоскостей в $P_{n}$, которые содержат $P_{r}$. Тогда множество $A_{n-r}^{*}=P_{n-r}^{*} \backslash P_{n-r-1}^{*}$ несет структуру $(n-r)$-мерного аффинного пространства и состоит из гиперплоскостей в $P_{n}$, которые содержат $P_{r-1}$ и не содержат $P_{r}$. Обозначим через $\mathscr{L}$ совокупность нуль-пар $(A, \Xi)$, для которых $A \in A_{r}$ и $\Xi \in A_{n-r}^{*}$, т. е. $\mathscr{L}=A_{r} \times A_{n-r}^{*} \subset P_{n} \times P_{n}^{*}$. Ясно, что $\mathscr{L}$ - гладкое $n$-мерное подмногообразие в $\mathfrak{N}$.

Пусть $\mathscr{F}(\mathscr{L})$ - подмногообразие в $\mathscr{F}(W)$, определяемое условиями

$$
P_{r}=\left\langle e_{0}, e_{1}, \ldots, e_{r}\right\rangle, \quad P_{r-1}=\left\langle e_{1}, e_{2}, \ldots, e_{r}\right\rangle
$$

Дифференциальные уравнения подмногообразия $\mathscr{F}(\mathscr{L})$ имеют вид

$$
\omega^{\alpha}=0, \quad \omega_{a}=0, \quad \omega_{a}^{\alpha}=0
$$

Здесь и далее $a, b, c, \ldots=1,2, \ldots, r ; \alpha, \beta, \gamma, \ldots=r+1, r+2, \ldots, n$. Легко проверить, что система (62) вполне интегрируема. Из последней серии уравнений этой системы следует, в частности, что подмногообразие $\mathscr{L}$ является вполне геодезическим. Кроме того, легко видеть, что при условии (62) форма $\omega^{i} \otimes \omega_{i}$ тождественно равна нулю. Следовательно, подмногообразие $\mathscr{L}$ вполне изотропно и лагранжево. 
При вложении $f_{s}$ в пространство $P_{K}$ параметрические уравнения подмногообразия $\mathscr{L}$ записываются в виде

$$
\begin{gathered}
X_{0}^{\alpha}=X_{a}^{0}=X_{a}^{\alpha}=X_{\beta}^{\alpha}=X_{b}^{a}=0 ; \\
\rho X_{0}^{0}=a^{0} \xi_{0} ; \quad \rho X_{0}^{a}=a^{a} \xi_{0} ; \quad \rho X_{\alpha}^{0}=a^{0} \xi_{\alpha} ; \quad \rho X_{\alpha}^{a}=a^{a} \xi_{\alpha} ; \\
a^{0} \neq 0 ; \quad \xi_{0} \neq 0 .
\end{gathered}
$$

Эта система определяет аффинную область $A_{r} \times A_{n-r}^{*}$ на многообразии Сегре $S(r, n-r) \subset \mathfrak{W} \subset P_{K}$. Эта область получается из многообразия Сегре $S(r, n-r)$ после удаления из последнего замкнутого подмножества $a^{0}=\xi_{0}=0$.

Итак, можно сформулировать

УТВЕРЖДЕНИЕ 2. Пусть $\mathscr{L}$ - множсество нуль-пар из примера 3. Тогда $\mathscr{L}$ - гладкое п-мерное подмногообразие в $\mathfrak{N}$, диффеоморфное аффинному пространству $A_{n} \equiv A_{r} \times A_{n-r}$. Подмногообразие $\mathscr{L}$ является лагранжевым, вполне геодезическим и вполне изотропным. Образ $f_{s}(\mathscr{L})$ многообразия $\mathscr{L}$ при вложении $f_{s}: \mathfrak{N} \rightarrow \mathfrak{W} \subset P_{K}$ есть аффинная область на многообразии Cerpe $S(r, n-r) \subset \mathfrak{W}$.

ТЕОРема 9. Пусть $\mathscr{L}$ - $n$-мерное вполне изотропное лагранжево подмногообразие на многообразии 以. Тогда $\mathscr{L}$ - либо плоскость из примеров 1,2 , либо аффинная область на многообразии Сегре, описанная в примере 3 . В любом случае $\mathscr{L}$ - вполне геодезическое подмногообразие.

ДокАЗАТЕльство. Пусть $\left\{\zeta_{i}=a_{i}+b_{i}=A_{i}^{j} v_{j}+A_{i j} v^{j}\right\}$ - базис касательного пространства к подмногообразию $\mathscr{L}$. Здесь $\left\{v_{i}\right\}-$ базис, дуальный для кобазиса $\left\{\omega^{i}\right\}$, а $\left\{v^{i}\right\}$ - базис, дуальный для кобазиса $\left\{\omega_{i}\right\}$. Условие изотропности и лагранжевости записывается в виде $\omega^{i}\left(\zeta_{j}\right) \otimes \omega_{i}\left(\zeta_{k}\right)=0$. Следовательно,

$$
\omega^{i}\left(\zeta_{j}\right) \otimes \omega_{i}\left(\zeta_{k}\right)=\omega^{i}\left(A_{j}^{d} v_{l}+A_{k l} v^{l}\right) \otimes \omega_{i}\left(A_{k}^{r} v_{r}+A_{k r} v^{r}\right)=A_{j}^{i} A_{k i}=0
$$

Итак,

$$
A_{j}^{i} A_{k i}=0
$$

Рассмотрим три случая.

1) Пусть $\operatorname{det}\left(A_{j}^{i}\right) \neq 0$. Тогда $A_{k i}=0$. Пусть $X=X^{i} \zeta_{i}=X^{i} A_{i}^{j} v_{j}-$ произвольный касательный вектор к $\mathscr{L}$. Тогда $\omega_{i}(X)=0$. Следовательно, подмногообразие $\mathscr{L}$ определяется на $\mathfrak{W}$ вполне интегрируемой системой Пфаффа (60). Таким образом, мы приходим к примеру 1.

2) Пусть $\operatorname{det}\left(A_{i j}\right) \neq 0$. Тогда $A_{k}^{i}=0$ и подмногообразие $\mathscr{L}$ определяется на $\mathfrak{W}$ системой Пфафффа (61). Следовательно, мы приходим к примеру 2.

3) Пусть $\operatorname{det}\left(A_{j}^{i}\right)=\operatorname{det}\left(A_{i j}\right)=0$. Предположим, что

$$
\operatorname{rank}\left\{a_{1}, a_{2}, \ldots, a_{n}\right\}=r
$$


Тогда

$$
\operatorname{rank}\left\{b_{1}, b_{2}, \ldots, b_{n}\right\}=n-r .
$$

При помощи перенумерации векторов $\left\{v_{i}\right\}$ и $\left\{a_{i}\right\}$ можно добиться того, чтобы вьполнялись условия: $\left\{a_{1}, a_{2}, \ldots, a_{r}\right\}$ - базис подпространства $\left\langle a_{1}, a_{2}, \ldots, a_{n}\right\rangle$ и $\left(A_{a}^{b}\right)$ - невырожденная $(r \times r)$-матрица, $a, b=1,2, \ldots, r$. Тождества (63) перепишутся в виде

$$
\begin{gathered}
A_{a}^{c} A_{b c}+A_{a}^{\alpha} A_{b \alpha}=0 ; \quad A_{a}^{c} A_{\beta c}+A_{a}^{\alpha} A_{\beta \alpha}=0 ; \\
A_{\beta}^{c} A_{b c}+A_{\beta}^{\alpha} A_{b \alpha}=0 ; \quad A_{\gamma}^{c} A_{\beta c}+A_{\gamma}^{\alpha} A_{\beta \alpha}=0 ; \\
a, b, c, l=1,2, \ldots, r ; \quad \alpha, \beta, \gamma=r+1, r+2, \ldots, n .
\end{gathered}
$$

Обозначим через $\left(\bar{A}_{a}^{b}\right)$ матрицу, обратную матрище $\left(A_{a}^{b}\right)$, иположим $a_{\alpha}=\lambda_{\alpha}^{a} a_{a}$, т. е. $A_{\alpha}^{j}=\lambda_{\alpha}^{a} A_{a}^{j}$. Тогда из последней системы тождеств найдем $A_{i a}=-\lambda_{a}^{\alpha} A_{i \alpha}$, где $\lambda_{a}^{\alpha}=\bar{A}_{a}^{b} A_{b}^{\alpha}$.

Пусть $\zeta=x^{i} \zeta_{i}-$ произвольный касательный вектор к подмногообразию $\mathscr{L}$. Имеем

$$
\zeta=\left(x^{a}+\lambda_{\alpha}^{a} x^{\alpha}\right) A_{a}^{j} v_{j}+x^{j} A_{j \alpha}\left(v^{\alpha}-\lambda_{b}^{\alpha} v^{b}\right) .
$$

Следовательно,

$$
\begin{array}{ll}
\omega^{a}(\zeta)=A_{b}^{a}\left(x^{b}+x^{\alpha} \lambda_{\alpha}^{b}\right), & \omega^{\alpha}(\zeta)=A_{b}^{\alpha}\left(x^{b}+x^{\beta} \lambda_{\beta}^{b}\right), \\
\omega_{a}(\zeta)=-x^{j} A_{j \alpha} \lambda_{a}^{\alpha}, & \omega_{\alpha}(\zeta)=-x^{j} A_{j \alpha} .
\end{array}
$$

Поэтому

$$
\omega^{\alpha}(\zeta)=\bar{A}_{a}^{b} A_{b}^{\alpha} \omega^{a}(\zeta)=\lambda_{a}^{\alpha} \omega^{a}(\zeta), \quad \omega_{a}(\zeta)=-\lambda_{a}^{\alpha} \omega_{\alpha}(\zeta)
$$

Итак, подмногообразие $\mathscr{L}$ определяется на $\mathfrak{W}$ системой дифференциальных уравнений

$$
\omega^{\alpha}=\lambda_{a}^{\alpha} \omega^{a}, \quad \omega_{a}=-\lambda_{a}^{\alpha} \omega_{\alpha}
$$

где $a=1,2, \ldots, r, \alpha=r+1, r+2, \ldots, n$ и формы $\omega^{a}, \omega_{\alpha}$ линейно независимы на $\mathscr{L}$.

Легко видеть, что при условии (64) форма $\omega^{i} \otimes \omega_{i}$ обрашается в нуль. Действительно,

$$
\omega^{i} \otimes \omega_{i}=\omega^{\alpha} \otimes \omega_{\alpha}+\omega^{a} \otimes \omega_{a}=\lambda_{a}^{\alpha} \omega^{a} \otimes \omega_{\alpha}+\omega^{a} \otimes\left(-\lambda_{a}^{\alpha} \omega_{\alpha}\right)=0 .
$$

Следовательно, если существует подмногообразие $\mathscr{L}$, определяемое системой (64), то оно будет вполне изотропным и лагранжевым.

Условие интегрируемости системы (64) имеет вид

$$
\begin{aligned}
& \left(d \lambda_{a}^{\alpha}+\lambda_{a}^{\beta} \theta_{\beta}^{\alpha}-\lambda_{b}^{\alpha} \theta_{a}^{b}-\omega_{a}^{\alpha}+\lambda_{a}^{\beta} \lambda_{b}^{\alpha} \theta_{\beta}^{b}\right) \wedge \omega_{\alpha}=0, \\
& \left(d \lambda_{a}^{\alpha}+\lambda_{a}^{\beta} \theta_{\beta}^{\alpha}-\lambda_{b}^{\alpha} \theta_{a}^{b}-\omega_{a}^{\alpha}+\lambda_{a}^{\beta} \lambda_{b}^{\alpha} \theta_{\beta}^{b}\right) \wedge \omega^{a}=0 .
\end{aligned}
$$


Отсюда, в силу линейной независимости 1-форм $\omega^{a}, \omega_{\alpha}$ находим

$$
d \lambda_{a}^{\alpha}+\lambda_{a}^{\beta} \theta_{\beta}^{\alpha}-\lambda_{b}^{\alpha} \theta_{a}^{b}-\omega_{a}^{\alpha}+\lambda_{a}^{\beta} \lambda_{b}^{\alpha} \theta_{\beta}^{b}=0 .
$$

Из уравнений (65) следует, в частности, что подмногообразие $\mathscr{L}$ является вполне геодезическим.

Рассмотрим допустимую замену базиса $\left\{e_{u}\right\} \in \mathscr{F}(W)$ :

$$
e_{0} \rightarrow e_{0}, \quad e_{\alpha} \rightarrow e_{\alpha}, \quad e_{a} \rightarrow e_{a}+\lambda_{a}^{\alpha} e_{\alpha}
$$

Тогда сопряженный базис $\left\{e^{u}\right\}=h\left(\left\{e_{u}\right\}\right)$ преобразуется следуюшим образом:

$$
e^{0} \rightarrow e^{0}, \quad e^{\alpha} \rightarrow e^{\alpha}-\lambda_{a}^{\alpha} e^{a}, \quad e^{a} \rightarrow e^{a} .
$$

В новом базисе величины $\lambda_{s}^{\alpha}$ будут равны нулю, а система уравнений (64), (65), определяющая подмногообразие $\mathscr{L}$, примет вид (62). Тем самым мы приходим к примеру 3.

СЛЕДСТВИЕ 2. Пусть $\Lambda$ - абстрактное множество всех подпространств $n$-мерного векторного пространства $\mathcal{V}$, включающее нулевое подпространство $\{0\}$ и само пространство $\mathcal{V}$. Тогда әлементы множества $\Lambda$ находятся в биективном соответствии с элементами множества всех $n$-мерных вполне изотропных лагранжевых подмногообразий, проходящих через фиксированную точку многообразия невырожденных нуль-пар. При әтом подпространствам $\mathcal{V}$ u $\{0\}$ соответствуют плоские образующие из примеров 1 и 2, а остальным подпространствам соответствуют аффинные области на многообразиях Сегре из примера 3.

ДокАЗАТЕЛЬСТво. Пусть $x=(\langle a\rangle,\langle\xi\rangle)$ - произвольная точка на многообразии $\mathfrak{N}$ невырожденных нуль-пар, $T_{x} \mathfrak{N}=(W /\langle a\rangle) \oplus\left(W^{*} /\langle\xi\rangle\right)$ - касательное пространство, $\mathscr{V}=W /\langle a\rangle-n$-мерное подпространство в $T_{x} \mathfrak{W}$, касательное к первой плоской образующей, П - произвольное подпространство в $\mathscr{V}$.

Если $\Pi=\mathscr{V}$, то сопоставим подпространству П лагранжево многообразие из примера 1, построенное при помощи гиперплоскости $\Xi_{0}=\langle\xi\rangle$.

Если $\Pi=\{0\}$, то сопоставим подпространству П лагранжево подмногообразие из примера 2 , построенное при помоши точки $A_{0}=\langle a\rangle$.

Пусть $\operatorname{dim} \Pi=r$, где $1 \leqslant r \leqslant n$. Сушествуют единственная $r$-мерная проективная плоскость $P_{r} \subset P_{n}$, проходящая через $\langle a\rangle$ в направлении П, и единственная $(r-1)$-мерная плоскость, по которой плоскость $P_{r}$ пересекается с гиперплоскостью $\langle\xi\rangle$. Так как $P_{r-1} \subset P_{r}$, то можно рассмотреть лагранжево подмногообразие $\mathscr{L}$, построенное в примере 3 при помощи плоскостей $P_{r}$ и $P_{r-1}$. Ясно, что это подмногообразие проходит через точку $x$.

Очевидно, что построенное соответствие биективно.

Условие теоремы 9 можно значительно ослабить, отказавшись от предположения лагранжевости. Оказьвается, что условие существования $n$-мерного вполне изотропного подмногообразия в многообразии невырожденных нуль-пар приводит к его лагранжевости. Следующая теорема дает полную классификацию вполне изотропных подмногообразий максимальной размерности. 
Teорема 10. Пусть $\mathscr{L}$ - $n$-мерное вполне изотропное подмногообразие на многообразии невырожденных нуль-пар $\mathfrak{W}$. Тогда $\mathscr{L}$ - лагранжсево подмногообразие, которое в силу теоремы 9 вполне геодезично и является либо плоскостью, описанной в примерах 1,2 , либо аффинной областью на многообразии Сегре, описанной в примере 3 .

ДокАЗАТЕльство. Пусть $\left\{\zeta_{i}=a_{i}+b_{i}=A_{i}^{j} v_{j}+A_{i j} v^{j}\right\}$ - базис касательного пространства к подмногообразию $\mathscr{L}$. Здесь, как и ранее, $\left\{v_{i}\right\}$ - базис, дуальньй кобазису $\left\{\omega^{i}\right\}$, а $\left\{v^{i}\right\}$ - базис, дуальный кобазису $\left\{\omega_{i}\right\}$. Условие полной изотропности $\omega^{i} \odot \omega_{i} \equiv 0$ запишется в виде

$$
A_{j}^{i} A_{k i}+A_{k}^{i} A_{j i}=0 .
$$

Как и ранее, возможны три случая.

1) Пусть $\operatorname{det}\left(A_{j}^{i}\right) \neq 0$. Тогда существует матрища $\left(\bar{A}_{j}^{i}\right)$, обратная для $\left(A_{j}^{i}\right)$. Пусть $\zeta=x^{i} \zeta_{i}=x^{i}\left(A_{i}^{j} v_{j}+A_{i j} v^{j}\right)$ - произвольный вектор, касательный к $\mathscr{L}$. Тогда

$$
\omega^{i}(\zeta)=x^{j} A_{j}^{i}, \quad \omega_{i}(\zeta)=x^{j} A_{j i}=A_{j i} \bar{A}_{k}^{j} \omega^{k}(\zeta) .
$$

Следовательно, $\omega_{i}=\lambda_{i k} \omega^{k}$, где $\lambda_{i k}=A_{j i} \bar{A}_{k}^{j}$. Из соотношения (66) находим, что $A_{i j}=-A_{i}^{l} \bar{A}_{j}^{k} A_{k l}$. Имеем

$$
\lambda_{(i k)}=A_{j(i} \bar{A}_{k)}^{j}=-\frac{1}{2}\left(A_{j}^{l} \bar{A}_{i}^{s} A_{s l} \bar{A}_{k}^{j}+A_{j}^{l} \bar{A}_{k}^{s} A_{s l} \bar{A}_{i}^{j}\right)=-A_{s(k} \bar{A}_{i)}^{s}=-\lambda_{(i k)} .
$$

Следовательно, $\lambda_{(i k)}=0$. Итак, подмногообразие $\mathscr{L}$ определяется на $\mathfrak{W}$ системой Пфаффа

$$
\omega_{i}=\lambda_{i j} \omega^{j}, \quad \text { где } \lambda_{(i j)}=0 .
$$

При этом 1-формы $\omega^{i}$ линейно независимы на $\mathscr{L}$, так как $\operatorname{dim} \mathscr{L}=n$.

Дифференцируя систему (67) внешним образом и используя лемму Картана, найдем

$$
\nabla \lambda_{i j}=\lambda_{i j k} \omega^{k}, \quad \text { где } \quad \lambda_{(i j) k}=\lambda_{i[j k]}=0 .
$$

Имеем

$$
\lambda_{i j k}=-\lambda_{j i k}=-\lambda_{j k i}=\lambda_{k j i}=\lambda_{k i j}=-\lambda_{i k j}=-\lambda_{i j k} .
$$

Следовательно, $\lambda_{i j k}=0$ и

$$
\nabla \lambda_{i j}=0
$$

Заметим, что из (68) следует, в частности, что $\mathscr{L}$ - вполне геодезическое подмногообразие. Дифференщируя (68), получим

$$
\lambda_{i k} \lambda_{j l}+\lambda_{i l} \lambda_{k j}+\lambda_{i j} \lambda_{k l}=0 .
$$

Отсюда при $k=i, l=j$ получим, что $\lambda_{i i} \lambda_{j j}+2\left(\lambda_{i j}\right)^{2}=0$. Поэтому в силу кососимметричности объекта $\lambda_{i j}$ найдем, что $\lambda_{i j}=0$. Система (67) перепишется так: $\omega_{i}=0$. Следовательно, $\mathscr{L}$ - плоскость, описанная в примере 1. 
2) Пусть $\operatorname{det}\left(A_{i j}\right) \neq 0$. Рассуждая в полной аналогии со случаем 1$)$, найдем, что подмногообразие $\mathscr{L}$ задается на $\mathfrak{W}$ системой Пфаффа $\omega^{i}=\lambda^{i j} \omega_{j}$, где $\lambda^{(i j)}=0$, а формы $\omega_{i}$ линейно независимы на $\mathscr{L}$. Однако, как и ранее, дифференцирование показывает, что подмногообразие $\mathscr{L}$ сушествует лишь в случае, когда $\lambda^{i j}=0$. Следовательно, $\mathscr{L}$ - плоскость из примера 2.

3) Пусть $\operatorname{det}\left(A_{j}^{i}\right)=\operatorname{det}\left(A_{i j}\right)=0$. Предположим, что

$$
\operatorname{rank}\left\{a_{1}, a_{2}, \ldots, a_{n}\right\}=r .
$$

Тогда

$$
\operatorname{rank}\left\{b_{1}, b_{2}, \ldots, b_{n}\right\}=n-r .
$$

При помощи перенумерации векторов $\left\{v_{i}\right\}$ и $\left\{a_{i}\right\}$ добьемся того, чтобы выполнялись условия: $\left\{a_{1}, a_{2}, \ldots, a_{r}\right\}$ - базис в $\left\langle a_{1}, a_{2}, \ldots, a_{n}\right\rangle$, а $\left(A_{a}^{b}\right)$ - невырожденная $(r \times r)$-матрица, где $a, b=1,2, \ldots, r$. Обозначим через $\left(\bar{A}_{a}^{b}\right)$ матрицу, обратную матрище $\left(A_{a}^{b}\right)$, и положим $a_{\alpha}=\lambda_{\alpha}^{a} a_{a}$, т. е. $A_{\alpha}^{j}=\lambda_{\alpha}^{a} A_{a}^{j}$. Используя тождества (66), найдем

$$
\begin{gathered}
A_{a b}=-\lambda_{b}^{\alpha} A_{a \alpha}-\bar{A}_{b}^{c} A_{a}^{d} A_{c d}-\bar{A}_{b}^{c} A_{a}^{\alpha} A_{c \alpha} ; \\
A_{\alpha b}=\lambda_{\alpha}^{c} A_{c b}+\lambda_{b}^{\beta} A_{\alpha \beta}-\lambda_{b}^{\beta} \lambda_{\alpha}^{c} A_{c \beta} ; \\
A_{a}^{b}\left(\lambda_{\alpha}^{a} A_{\beta b}+\lambda_{\beta}^{a} A_{\alpha b}\right)=-A_{a}^{\gamma}\left(\lambda_{\alpha}^{a} A_{\beta \gamma}+\lambda_{\beta}^{a} A_{\alpha \gamma}\right) ; \\
\lambda_{\alpha}^{a}=\bar{A}_{b}^{a} A_{\alpha}^{b} ; \quad \lambda_{a}^{\alpha}=\bar{A}_{a}^{b} A_{b}^{\alpha} ; \\
a, b, c, l=1,2, \ldots, r ; \quad \alpha, \beta, \gamma=r+1, r+2, \ldots, n .
\end{gathered}
$$

Используя эти соотношения, несложно проверить, что для произвольного вектора $\zeta=x^{i} \zeta_{i}$, касательного к подмногообразию $\mathscr{L}$, имеют место равенства

$$
\omega^{\alpha}(\zeta)=\lambda_{a}^{\alpha} \omega^{a}(\zeta), \quad \omega_{a}(\zeta)=C_{a b} \omega^{b}(\zeta)-\lambda_{a}^{\alpha} \omega_{\alpha}(\zeta),
$$

где $C_{a b}=\bar{A}_{b}^{c}\left(A_{c a}+A_{c \beta} \lambda_{a}^{\beta}\right)$. Так как

$$
\begin{aligned}
2 C_{(a b)}= & \bar{A}_{b}^{c}\left(A_{c a}+A_{c \beta} \lambda_{a}^{\beta}\right)+\bar{A}_{a}^{c}\left(A_{c b}+A_{c \beta} \lambda_{b}^{\beta}\right) \\
= & \bar{A}_{a}^{d}\left(-\lambda_{b}^{\alpha} A_{d \alpha}-\bar{A}_{b}^{p} A_{d}^{c} A_{p c}-\bar{A}_{b}^{p} A_{d}^{\alpha} A_{p \alpha}+\bar{A}_{b}^{c} A_{c \alpha} A_{d}^{\alpha}\right) \\
& +\bar{A}_{b}^{d}\left(-\lambda_{a}^{\alpha} A_{d \alpha}-\bar{A}_{a}^{p} A_{d}^{c} A_{p c}-\bar{A}_{a}^{p} A_{d}^{\alpha} A_{p \alpha}+\bar{A}_{a}^{c} A_{c \alpha} A_{d}^{\alpha}\right) \\
= & \bar{A}_{a}^{d}\left(-\lambda_{b}^{\alpha} A_{d \alpha}-\bar{A}_{b}^{p} A_{d}^{c} A_{p c}\right)+\bar{A}_{b}^{d}\left(-\lambda_{a}^{\alpha} A_{d \alpha}-\bar{A}_{a}^{p} A_{d}^{c} A_{p c}\right) \\
= & -\bar{A}_{a}^{d} \lambda_{b}^{\alpha} A_{d \alpha}-\bar{A}_{b}^{p} A_{p a}-\bar{A}_{b}^{d} \lambda_{a}^{\alpha} A_{d \alpha}-\bar{A}_{a}^{p} A_{p b} \\
= & -\bar{A}_{a}^{d}\left(\lambda_{b}^{\alpha} A_{d \alpha}+A_{d b}\right)-\bar{A}_{b}^{p}\left(A_{p a}+\lambda_{a}^{\alpha} A_{p \alpha}\right)=-2 C_{(a b)},
\end{aligned}
$$

то $C_{(a b)}=0$. Итак, подмногообразие $\mathscr{L} \subset \mathfrak{W}$ определяется системой дифференциальньхх уравнений

$$
\omega^{\alpha}=\lambda_{a}^{\alpha} \omega^{a}, \quad \omega_{a}=C_{a b} \omega^{b}-\lambda_{a}^{\alpha} \omega_{\alpha}
$$


где $C_{(a b)}=0$, а формы $\omega^{a}$ и $\omega_{\alpha}$ линейно независимы на $\mathscr{L}$. Дифференцирование системы (69) и использование леммы Картана приводит к соотношениям

$$
\begin{gathered}
d \lambda_{a}^{\alpha}-\lambda_{b}^{\alpha} \theta_{a}^{b}-\lambda_{a}^{\beta} \theta_{\beta}^{\alpha}+\theta_{a}^{\alpha}-\lambda_{b}^{\alpha} \lambda_{a}^{\beta} \theta_{\beta}^{b}=\lambda_{a b}^{\alpha} \omega^{b}, \\
d C_{a b}-C_{a c} \theta_{b}^{c}-C_{c b} \theta_{a}^{c}+2 C_{c[a} \lambda_{b]}^{\beta} \theta_{\beta}^{c}=C_{a b c} \omega^{c}-\lambda_{a b}^{\beta} \omega_{\beta}, \\
\lambda_{[a b]}^{\beta}=C_{(a b) c}=C_{a[b c]}=0 .
\end{gathered}
$$

Из соотношений (72) находим

$$
C_{a b c}=-C_{b a c}=-C_{b c a}=C_{c b a}=C_{c a b}=-C_{a c b}=-C_{a b c} .
$$

Следовательно, $C_{a b c}=0$. Кроме того, применяя операцию симметризации по индексам $a$ и $b$ к соотношению (71), найдем, что $\lambda_{a b}^{\beta}=0$. Поэтому система $(70),(71)$ примет вид

$$
\begin{aligned}
& d \lambda_{a}^{\alpha}-\lambda_{b}^{\alpha} \theta_{a}^{b}-\lambda_{a}^{\beta} \theta_{\beta}^{\alpha}+\theta_{a}^{\alpha}-\lambda_{b}^{\alpha} \lambda_{a}^{\beta} \theta_{\beta}^{b}=0 \\
& d C_{a b}-C_{a c} \theta_{b}^{c}-C_{c b} \theta_{a}^{c}+2 C_{c[a} \lambda_{b]}^{\beta} \theta_{\beta}^{c}=0 .
\end{aligned}
$$

Заметим, что из системы (73), (74), в частности, следует, что подмногообразие $\mathscr{L}$ является вполне геодезическим. Дифференцируя (73) и (74), получим

$$
C_{a b} C_{c d}+C_{a[c} C_{d] b}=0
$$

Полагая в этом тождестве $c=a$ и $d=b$, найдем, что $C_{a b}=0$.

Итак, система дифференщиальных уравнений (69), определяюшая вполне изотропное подмногообразие $\mathscr{L}$, примет вид

$$
\omega^{\alpha}=\lambda_{a}^{\alpha} \omega^{a}, \quad \omega_{a}=-\lambda_{a}^{\alpha} \omega_{\alpha}
$$

где формы $\omega^{a}$ и $\omega_{\alpha}$ линейно независимы на $\mathscr{L}$. Мы пришли к системе (64). Повторяя далее рассуждения из доказательства теоремы 9, приходим к выводу, что подмногообразие $\mathscr{L}$ является аффинной областью на многообразии Сегре, описанной в примере 3 .

ПРИмеР 4. Зафиксируем на $P_{n}$ произвольную гиперквадрику $Q$. Пусть $P_{n} \backslash Q-$ множество точек, не лежаших на $Q$. Для каждой точки $A \in P_{n} \backslash Q$ существует единственная гиперплоскость $\Xi$, полярная точке $A$ относительно $Q$. Так как $A \notin Q$, то $A \notin \Xi$. Пусть $\mathscr{L}$ - совокупность нуль-пар $(A, \Xi)$, для которых $A \in P_{n} \backslash Q$ полюс для $\Xi$ относительно $Q$. Ясно, что $\mathscr{L}$ - гладкое $n$-мерное подмногообразие в $N$, диффеоморфное $P_{n} \backslash Q$.

Заметим, что многообразие $\mathscr{L}$ может быть несвязным. Например, если $Q$ задается в $W$ квадратичной формой сигнатуры $(1, n)$, то $P_{n} \backslash Q$ - гиперболическое 
пространство, состоящее из двух компонент связности. Одна из компонент - пространство Лобачевского (внутренняя область относительно $Q$ ), а вторая - область идеальных точек гиперболического пространства (внешняя область относительно $Q)$.

Пусть относительно фиксированного базиса $\left\{\varepsilon_{u}\right\} \in \mathscr{F}(W)$ квадрика $Q$ определяется уравнением

$$
Q_{u v} X^{u} X^{v}=0
$$

При поляритете относительно $Q$ точке $A$ с однородными координатами $\left\{a^{u}\right\}$ соответствует гиперплоскость $\Xi$ с тангенциальными координатами $\left\{\xi_{u}\right\}$, где $\xi_{u}=$ $Q_{u v} a^{v}$. Поэтому при вложении $f_{s}: \mathfrak{N} \rightarrow \mathfrak{W} \subset P_{K}$ подмногообразие $\mathscr{L}$ будет задаваться в $P_{K}$ системой параметрических уравнений

$$
\rho X_{u}^{v}=Q_{v w} a^{u} a^{w}
$$

В векторной форме эта система переписывается следующим образом:

$$
\rho X=Q_{v w} a^{u} a^{w} \varepsilon_{u} \otimes \varepsilon^{v}
$$

Но последнее уравнение можно записать в виде

$$
\rho X=a^{u} a^{w} E_{u w}
$$

где $E_{u w}=Q_{v(w} \varepsilon_{u)} \otimes \varepsilon^{v}$ и $Q_{u v} a^{u} a^{v} \neq 0$. Пусть ранг системы тензоров $\left\{E_{u w}\right\}$ максимален, т. е. равен $\frac{n(n+3)}{2}+1$. Тогда $\mathscr{L}$ - открытое подмногообразие на многообразии Веронезе $\mathscr{B}$, вложенном в проективное пространство размерности $\frac{n(n+3)}{2}$. Это подмногообразие получено из многообразия Веронезе $\mathscr{B}$ удалением замкнутого подмножества $Q_{u v} a^{u} a^{v}=0$. Если ранг системы тензоров $\left\{E_{u w}\right\}$ меньше $\frac{n(n+3)}{2}+1$, то $\mathscr{L}$ является образом многообразия Веронезе $\mathscr{B}$ (точнее, его открытого подмногообразия) при центральном проектировании на некоторое подпространство из многомерного центра проекции.

Пусть $\mathscr{F}(\mathscr{L})$ - подмногообразие базисов из $\mathscr{F}(W)$, для каждого из которых точка $A=p\left(e_{0}\right)$ и гиперплоскость $\Xi=p\left(e^{0}\right)$ находятся в полярном соответствии относительно квадрики $Q$. В любом репере из $\mathscr{F}(\mathscr{L})$ уравнение $(75)$ записьвается в виде

$$
X^{0} X^{0}-A_{i j} X^{i} X^{j}, \quad A_{[i j]}=0
$$

а условие инвариантности этой квадрики задается системой дифференщиальных уравнений

$$
\begin{gathered}
\omega_{i}-A_{i j} \omega^{j}=0, \quad A_{[i j]}=0 \\
d A_{i j}-A_{j k} \omega_{i}^{k}-A_{i k} \omega_{j}^{k}+2 A_{i j} \omega_{0}^{0}=0 .
\end{gathered}
$$


Легко проверить, что система $(77),(78)$ вполне интегрируема на $\mathfrak{W}$. Пусть $x-$ произвольная точка на $\mathfrak{W}$, а $A_{i j} v^{i} v^{j}$ - произвольная квадратичная форма, заданная на $n$-мерном векторном пространстве $\mathfrak{W}$. Тогда существует единственное подмногообразие $\mathscr{L}$, проходящее через $x$ и удовлетворяюшее системе дифференщиальных уравнений (77), (78). В силу (77) симплектическая форма $\Omega=\omega^{i} \wedge \omega_{i}$ на подмногообразии $\mathscr{L}$ обращается в нуль, поэтому $\mathscr{L}$ - лагранжево подмногообразие. Более того, из системы (78) непосредственно следует, что $\mathscr{L}$ - вполне геодезическое подмногообразие.

ПРИмеР 5. Рассмотрим конструкцию, двойственную примеру 4 . Пусть $Q-$ гиперквадрика в пространстве $P_{n}^{*}$, а $\mathscr{L}$ - совокупность нуль-пар $(A, \Xi)$, для которых $\Xi \in P_{n}^{*} \backslash Q$, а точка $A$ - полюс для $\Xi$ относительно $Q$. Тогда $\mathscr{L}$ - гладкое $n$-мерное подмногообразие в $\mathfrak{N}$, диффеоморфное $P_{n}^{*} \backslash Q$. Подмногообразие $\mathscr{L}$ задается на $\mathfrak{N}$ системой дифференщиальных уравнений

$$
\omega^{i}-A^{i j} \omega_{j}=0, \quad A^{[i j]}=0, \quad d A^{i j}+A^{j k} \omega_{j}^{i}+A^{i k} \omega_{k}^{j}-2 A^{i j} \omega_{0}^{0}=0
$$

и удовлетворяет всем свойствам подмногообразия из примера 4.

Таким образом, справедливо

УТВЕРЖДЕНИЕ 3. Пусть $\mathscr{L}$ - множество нуль-пар из примера 4 или из примера 5. Тогда $\mathscr{L}$ - гладкое $n$-мерное вполне геодезическое лагранжево подмногообразие в $\mathfrak{N}$. Образ $f_{s}(\mathscr{L})$ многообразия $\mathscr{L}$ при вложении $f_{s}: \mathfrak{N} \rightarrow$ $\mathfrak{W} \subset P_{K}$ является либо открытым подмногообразием на многообразии Веронезе $\mathscr{B}$, либо его образом при иентральном проектировании из многомерного иентра. Лагранжевы подмногообразия из примера 4 или из примера 5, проходящие через фиксированную точку многообразия $\mathfrak{N}$, находятся в биективном соответствии с квадратичными формами на п-мерном векторном пространстве.

СЛЕДСТВИЕ 3. Пусть $Q-$ мнимая квадрика на $P_{n}\left(\right.$ на $\left.P_{n}^{*}\right)$. Тогда в надлежашем репере $Q_{u v}=\delta_{u v}\left(Q^{u v}=\delta^{u v}\right)$. Следовательно, параметрические уравнения подмногообразия $\mathscr{L}$ из примера 4 (из примера 5) запишутся в виде $\rho X_{u}^{v}=a^{u} a^{v} \quad\left(\rho X_{u}^{v}=\xi_{u} \xi_{v}\right)$. Поэтому $\mathscr{L}$ - это в точности многообразие Веронезе, диффеоморфное $P_{n}$. В частности, $\mathscr{L}$ компактно.

Напомним, что само многообразие невырожденных нуль-пар некомпактно.

ПримеР 6 . Пусть $P_{r}$ - фиксированная $r$-плоскость в проективном пространстве $P_{n}$, и пусть $Q$ - гиперквадрика в $P_{r}$. Для каждой точки $A \in P_{r} \backslash Q$ существует единственная $(r-1)$-плоскость $\pi \subset P_{r}$, полярная точке $A$ относительно квадрики $Q$. Семейство гиперплоскостей в $P_{n}$, пересекающих $P_{r}$ по плоскости $\pi$ и не содержаших $P_{r}$, зависит от $n-r$ параметров и является $(n-r)$-мерным аффинным пространством. Обозначим это семейство гиперплоскостей через $A_{n-r}^{*}(A)$. Рассмотрим множество $\mathscr{L}$ невырожденных нуль-пар $(A, \Xi)$, для которых $A \in P_{r} \backslash Q$ и $\Xi \in A_{n-r}^{*}(A)$. Несложно доказать, что $\mathscr{L}$ - гладкое $n$-мерное подмногообразие в многообразии невырожденных нуль-пар $\mathfrak{N}$. 
Пусть $\mathscr{F}(\mathscr{L})$ - подмногообразие реперов из $\mathscr{F}(W)$, определяемых следуюшим условием: $\left\langle e_{0}, e_{1}, \ldots, e_{r}\right\rangle=P_{r}-$ фиксированная плоскость. Тогда на $\mathscr{F}(\mathscr{L})$ имеем

$$
\omega^{\alpha}=0, \quad \omega_{a}^{\alpha}=0 .
$$

Пусть относительно базиса $\left\{e_{a}\right\}$ уравнение квадрики $Q$ имеет вид

$$
x^{0} x^{0}-A_{a b} x^{a} x^{b}=0, \quad A_{[a b]}=0 .
$$

Условие инвариантности квадрики $Q$ в подпространстве $P_{r}$ записьвается в виде

$$
\omega_{a}=A_{a b} \omega^{b}, \quad d A_{a b}-A_{c b} \omega_{a}^{c}-A_{a c} \omega_{b}^{c}+2 A_{a b} \omega_{0}^{0}=0 .
$$

Несложно показать, что формы $\omega^{a}$ и $\omega_{\alpha}$ линейно независимы на подмногообразии $\mathscr{L} \subset \mathfrak{W}$. Само подмногообразие $\mathscr{L}$ определяется на $\mathfrak{W}$ системой дифференциальных уравнений

$$
\begin{gathered}
\omega^{\alpha}=0, \quad \omega_{a}^{\alpha}=0, \quad \omega_{a}=A_{a b} \omega^{b}, \\
d A_{a b}-A_{c b} \omega_{a}^{c}-A_{a c} \omega_{b}^{c}+2 A_{a b} \omega_{0}^{0}=0 .
\end{gathered}
$$

Ясно, что на $\mathscr{L}$ симплектическая форма $\Omega=\omega^{i} \wedge \omega_{i}$ тождественно обрашается в нуль. Следовательно, $\mathscr{L}$ - лагранжево подмногообразие. Более того, из последней системы следует, что подмногообразие $\mathscr{L}$ вполне геодезично.

При вложении $f_{s}$ в пространство $P_{K}$ параметрические уравнения подмногообразия $\mathscr{L}$ в надлежашем репере записываются в виде

$$
\begin{array}{ccc}
\rho X_{0}^{0}=a^{0} a^{0} ; & \rho X_{a}^{0}=-a^{0} A_{a b} a^{b} ; & \rho X_{\alpha}^{0}=a^{0} \xi_{\alpha} ; \\
\rho X_{0}^{a}=a^{a} a^{0} ; & \rho X_{b}^{a}=-a^{a} A_{b c} a^{c} ; & \rho X_{\alpha}^{a}=a^{a} \xi_{\alpha} ; \\
X_{0}^{\alpha}=X_{a}^{\alpha}=X_{\beta}^{\alpha}=0 . &
\end{array}
$$

Пусть $y^{0}=a^{0}, y^{a}=a^{a}, y^{\alpha}=\xi_{\alpha}$. Рассмотрим совокупность тензоров из $W \otimes W^{*}:$

$$
\begin{aligned}
& E_{00}=e_{0}^{0} ; \quad E_{0 a}=E_{a 0}=\frac{1}{2}\left(-A_{a b} e_{0}^{b}+e_{a}^{0}\right) ; \quad E_{0 \alpha}=E_{\alpha 0}=\frac{1}{2} e_{0}^{\alpha} ; \\
& E_{a b}=E_{b a}=-A_{c(a} e_{b)}^{c} ; \quad E_{a \alpha}=E_{\alpha a}=\frac{1}{2} e_{a}^{\alpha} ; \quad E_{\alpha \beta}=E_{\beta \alpha}=0 .
\end{aligned}
$$

Теперь уравнение подмногообразия $\mathscr{L}$ можно записать в векторной форме:

$$
\rho X=y^{u} y^{v} E_{u v}
$$

где $y^{0} y^{0}-A_{a b} y^{a} y^{b} \neq 0 ; u, v=0,1, \ldots, n ; \quad a, b=1,2, \ldots, r$. Так как тензоры $E_{u v}$ линейно независимы, то это уравнение определяет образ многообразия Веронезе $\mathscr{B}$ при центральном проектировании на подпространство, порожденное линейной оболочкой тензоров $E_{u v}$, из некоторого многомерного центра. Лагранжево подмногообразие $\mathscr{L}$ является открытым подмногообразием в $\mathscr{B}$ и получается из последнего удалением замкнутого подмножества, которое задается уравнением $y^{0} y^{0}-A_{a b} y^{a} y^{b}=0$.

Итак, имеет место 
УТВЕРЖДЕНИЕ 4. Пусть $\mathscr{L}$ - множество нуль-пар из примера 6. Тогда $\mathscr{L}$ - гладкое $n$-мерное вполне геодезическое лагранжево подмногообразие в $\mathfrak{N}$. Подмногообразие $f_{s}(\mathscr{L}) \subset \mathfrak{W} \subset P_{K}$ является образом открытого подмногообразия на многообразии Веронезе $\mathscr{B}$ при центральном проектировании из многомерного иентра.

Следуюшая теорема дает полную классификацию вполне геодезических лагранжевых подмногообразий максимальной размерности многообразия $\mathbb{R} P_{n} \diamond \mathbb{R} P_{n}$.

Теорема 11. Пусть $\mathscr{L}$ - n-мерное вполне геодезическое лагранжево подмногообразие на многообразии невырожденных нуль-пар $\mathfrak{N}$. Тогда $\mathscr{L}$ - либо вполне изотропное подмногообразие из теоремы 10, либо одно из подмногообразий, описанных в примерах 4-6.

ДокАЗАТЕЛЬСТво. Пусть векторы $\zeta_{i}=a_{i}+b_{i}=A_{i}^{j} v_{j}+A_{i j} v^{j}$ составляют базис касательного пространства к подмногообразию $\mathscr{L}$. Здесь, как и ранее, $\left\{v_{i}\right\}-$ базис, дуальный кобазису $\left\{\omega^{i}\right\}$, а $\left\{v^{i}\right\}$ - базис, дуальньй кобазису $\left\{\omega_{i}\right\}$. Условие лагранжевости $\omega^{k}\left(\zeta_{i}\right) \wedge \omega_{k}\left(\zeta_{j}\right) \equiv 0$ записывается в виде

$$
A_{j}^{i} A_{k i}-A_{k}^{i} A_{j i}=0 .
$$

Как и ранее, рассмотрим три случая.

1) Пусть $\operatorname{det}\left(A_{j}^{i}\right) \neq 0$. Тогда сушествует матрища $\left(\bar{A}_{j}^{i}\right)$, обратная матрище $\left(A_{j}^{i}\right)$. Пусть $\zeta=x^{i} \zeta_{i}=x^{i}\left(A_{i}^{j} v_{j}+A_{i j} v^{j}\right)$ - произвольный вектор, касательный к $\mathscr{L}$. Тогда

$$
\omega^{i}(\zeta)=x^{j} A_{j}^{i}, \quad \omega_{i}(\zeta)=x^{j} A_{j i}=A_{j i} \bar{A}_{k}^{j} \omega^{k}(\zeta)
$$

Следовательно, $\omega_{i}=\lambda_{i k} \omega^{k}$, где $\lambda_{i k}=A_{j i} \bar{A}_{k}^{j}$. Из (81) находим, что $A_{i j}=$ $A_{i}^{l} \bar{A}_{j}^{k} A_{k l}$. Имеем, далее,

$$
2 \lambda_{[i j]}=\bar{A}_{j}^{k} A_{k i}-\bar{A}_{i}^{k} A_{k j}=\bar{A}_{j}^{k} A_{k i}-\bar{A}_{i}^{k} \bar{A}_{j}^{s} A_{k}^{l} A_{s l}=\bar{A}_{j}^{k} A_{k i}-\bar{A}_{j}^{s} A_{s i}=0 .
$$

Следовательно, $\lambda_{[i j]}=0$. Итак, подмногообразие $\mathscr{L}$ определяется на $\mathfrak{W}$ системой Пфаффа

$$
\omega_{i}=\lambda_{i j} \omega^{j}, \quad \text { где } \quad \lambda_{[i j]}=0 .
$$

При этом 1-формы $\omega^{i}$ линейно независимы на $\mathscr{L}$, так как $\operatorname{dim} \mathscr{L}=n$.

Если $\lambda_{i j}=0$, то система (82) примет вид (60) и будет определять лагранжево вполне изотропное подмногообразие из примера 1 , т. е. $n$-мерную плоскую образующую многообразия $\mathfrak{M}$.

Пусть $\operatorname{rank}\left(\lambda_{i j}\right)>0$. Дифференцируя систему (82) внешним образом и используя лемму Картана, найдем

$$
\nabla \lambda_{i j}=\lambda_{i j k} \omega^{k}
$$


где $\lambda_{i j k}$ симметричны по всем индексам. Так как по условию теоремы подмногообразие $\mathscr{L}$ является вполне геодезическим, то $\lambda_{i j k}=0$. Следовательно, подмногообразие $\mathscr{L}$ определяется на $\mathfrak{N}$ системой дифференциальных уравнений

$$
\begin{gathered}
\omega_{i}-\lambda_{i j} \omega^{j}=0, \quad \lambda_{[i j]}=0, \\
d \lambda_{i j}-\lambda_{j k} \omega_{i}^{j}-\lambda_{i k} \omega_{j}^{k}+2 \lambda_{i j} \omega_{0}^{0}=0 .
\end{gathered}
$$

Таким образом, приходим к системе $(77),(78)$ которая вполне интегрируема и определяет лагранжево подмногообразие из примера 4.

$2)$ Пусть $\operatorname{det}\left(A_{i j}\right) \neq 0$. Этот случай двойствен случаю 1 . Подмногообразие $\mathscr{L}$ будет определяться системой Пфаффа

$$
\begin{gathered}
\omega^{i}-\lambda^{i j} \omega_{j}=0, \quad \lambda^{[i j]}=0, \\
d \lambda^{i j}+\lambda^{j k} \omega_{j}^{i}+\lambda^{i k} \omega_{k}^{j}-2 \lambda^{i j} \omega_{0}^{0}=0 .
\end{gathered}
$$

При $\lambda^{i j}=0$ приходим к примеру 2, а при $\operatorname{rank}\left(\lambda_{i j}\right)>0$ - к примеру 5 .

3) Допустим теперь, что $\operatorname{det}\left(A_{j}^{i}\right)=\operatorname{det}\left(A_{i j}\right)=0$. Предположим, что $\operatorname{rank}\left\{a_{1}\right.$, $\left.a_{2}, \ldots, a_{n}\right\}=\operatorname{rank}\left(A_{i}^{j}\right)=r$. Тогда $\operatorname{rank}\left\{b_{1}, b_{2}, \ldots, b_{n}\right\}=n-r$. Без ограничения обшности можно считать, что $\left\{a_{1}, a_{2}, \ldots, a_{r}\right\}$ - базис подпространства $\left\langle a_{1}, a_{2}, \ldots, a_{n}\right\rangle$ и $\left(A_{a}^{b}\right)$ - невырожденная $(r \times r)$-матрица, где $a, b=1,2, \ldots, r$. Обозначим через $\left(\bar{A}_{a}^{b}\right)$ матрицу, обратную матрице $\left(A_{a}^{b}\right)$, и положим $a_{\alpha}=\lambda_{\alpha}^{a} a_{a}$, т. е. $A_{\alpha}^{j}=\lambda_{\alpha}^{a} A_{a}^{j}$. Используя тождества (81), найдем

$$
\begin{gathered}
A_{a}^{c} A_{b c}+A_{a}^{\gamma} A_{b \gamma}=A_{b}^{c} A_{a c}+A_{b}^{\gamma} A_{a \gamma} ; \\
A_{a}^{c} A_{\beta c}+A_{a}^{\gamma} A_{\beta \gamma}=A_{\beta}^{c} A_{a c}+A_{\beta}^{\gamma} A_{a \gamma} ; \\
A_{\alpha}^{c} A_{\beta c}+A_{\alpha}^{\gamma} A_{\beta \gamma}=A_{\beta}^{c} A_{\alpha c}+A_{\beta}^{\gamma} A_{\alpha \gamma} ; \\
a, b, c, l=1,2, \ldots, r ; \quad \alpha, \beta, \gamma=r+1, r+2, \ldots, n .
\end{gathered}
$$

Пусть $\zeta=x^{i} \zeta_{i}-$ произвольный касательный вектор к подмногообразию $\mathscr{L}$. Тогда

$$
\begin{array}{ll}
\omega^{a}(\zeta)=A_{b}^{a}\left(x^{b}+x^{\beta} \lambda_{\beta}^{b}\right) ; & \omega^{\alpha}(\zeta)=A_{b}^{\alpha}\left(x^{b}+x^{\beta} \lambda_{\beta}^{b}\right) ; \\
\omega_{a}(\zeta)=x^{i} A_{i a} ; & \omega_{\alpha}(\zeta)=x^{i} A_{i} \alpha .
\end{array}
$$

Из соотношений (83)-(85) находим, что

$$
\omega^{\alpha}(\zeta)=\lambda_{a}^{\alpha} \omega^{a}(\zeta), \quad \omega_{a}(\zeta)=-\lambda_{a}^{\alpha} \omega^{\alpha}(\zeta)+C_{a b} \omega^{b}(\zeta),
$$

где $\lambda_{a}^{\alpha}=A_{b}^{\alpha} \bar{A}_{a}^{b}$ и $C_{a b}=\bar{A}_{a}^{d} A_{b}^{c} A_{d j} A_{c}^{j}$. Используя тождества (83), несложно доказать, что $C_{[a b]}=0$. Поэтому подмногообразие $\mathscr{L}$ будет определяться на $\mathfrak{N}$ системой дифференциальных уравнений

$$
\omega^{\alpha}=\lambda_{a}^{\alpha} \omega^{a}, \quad \omega_{a}=-\lambda_{a}^{\alpha} \omega^{\alpha}+C_{a b} \omega^{b}
$$


где $C_{[a b]}=0$, а формы $\omega^{a}$ и $\omega_{\alpha}$ линейно независимы на $\mathscr{L}$. Дифференщируя систему (86) и используя лемму Картана, найдем

$$
\begin{gathered}
d \lambda_{a}^{\alpha}-\lambda_{b}^{\alpha} \theta_{a}^{b}-\lambda_{a}^{\beta} \theta_{\beta}^{\alpha}+\theta_{a}^{\alpha}-\lambda_{b}^{\alpha} \lambda_{a}^{\beta} \theta_{\beta}^{b}=\lambda_{a b}^{\alpha} \omega^{b}, \\
d C_{a b}-C_{a c} \theta_{b}^{c}-C_{c b} \theta_{a}^{c}-2 C_{c(a} \lambda_{b)}^{\beta} \theta_{\beta}^{c}=C_{a b c} \omega^{c}-\lambda_{a b}^{\beta} \omega_{\beta},
\end{gathered}
$$

где $\lambda_{[a b]}^{\beta}=0$, a $C_{a b c}$ симметричны по всем индексам. Так как $\mathscr{L}$ - вполне геодезическое подмногообразие, то $\lambda_{a b}^{\beta}=C_{a b c}=0$. Следовательно,

$$
\begin{array}{r}
d \lambda_{a}^{\alpha}-\lambda_{b}^{\alpha} \theta_{a}^{b}-\lambda_{a}^{\beta} \theta_{\beta}^{\alpha}+\theta_{a}^{\alpha}-\lambda_{b}^{\alpha} \lambda_{a}^{\beta} \theta_{\beta}^{b}=0, \\
d C_{a b}-C_{a c} \theta_{b}^{c}-C_{c b} \theta_{a}^{c}-2 C_{c(a} \lambda_{b)}^{\beta} \theta_{\beta}^{c}=0 .
\end{array}
$$

Рассмотрим допустимую замену базиса:

$$
e_{0} \rightarrow e_{0}, \quad e_{\alpha} \rightarrow e_{\alpha}, \quad e_{a} \rightarrow e_{a}+\lambda_{a}^{\alpha} e_{\alpha}
$$

В новом репере величины $\lambda_{a}^{\alpha}$ станут равны нулю, а система дифференциальных уравнений (86)-(88), определяющая подмногообразие $\mathscr{L}$, примет вид

$$
\omega^{\alpha}=0, \quad \omega_{a}=C_{a b} \omega^{b}, \quad \omega_{a}^{\alpha}=0, \quad d C_{a b}-C_{a c} \theta_{b}^{c}-C_{c b} \theta_{a}^{c}=0
$$

Система (89) вполне интегрируема на $\mathfrak{N}$. Если $C_{a b}=0$, то приходим к системе (62), определяющей лагранжево подмногообразие из примера 3 . В случае, когда $\operatorname{rank}\left(C_{a b}\right)>0$, система (89) совпадает с (80) и определяет лагранжево подмногообразие из примера 6.

Итак, примеры 1-6 исчерпывают все вполне геодезические лагранжевы подмногообразия максимальной размерности на многообразии невырожденных нуль-пар. Количество таких подмногообразий, проходящих через каждую точку многообразия нуль-пар, может быть охарактеризовано следующим образом.

СЛЕДСТВИЕ 4. Пусть $\Lambda_{Q}$ - абстрактное множсество всех квадратичных форм на произвольных подпространствах п-мерного векторного пространства $\mathscr{V}$. Тогда әлементы множества $\Lambda_{Q}$ находятся в биективном соответствии с әлементами множсества всех $n$-мерных вполне геодезических лагранжевых подмногообразий, проходящих через фиксированную точку многообразия невырожденных нуль-пар. При этом подмножеству $\Lambda \subset \Lambda_{Q}$, состоящему из нулевых квадратичных форм на всех подпространствах пространства $\mathscr{V}$, соответствует подкласс вполне изотропных подмногообразий, проходящих через эту точку. 
2.6. Четырехмерный случай. Пусть теперь $\mathfrak{N}$-многообразие невырожденных нуль-пар на проективной плоскости $P_{2}$. Тогда $\mathfrak{N}$ - 4 -мерное псевдориманово многообразие с метрикой $\Psi=\omega^{1} \odot \omega_{1}+\omega^{2} \odot \omega_{2}$ сигнатуры $(2,2)$. Рассмотрим $C O(2,2)$-структуру, порожденную метрикой $\Psi$, т. е. псевдоконформную структуру сигнатуры $(2,2)$. Известно (см., например, [12]), что эта структура будет конформно полуплоской. Этот факт непосредственно связан с геометрией двумерных вполне изотропных подмногообразий на $\mathfrak{N}$.

В каждой точке $x 4$-мерного псевдориманова многообразия $M$ с метрикой $\Psi=$ $\omega^{1} \odot \omega_{1}+\omega^{2} \odot \omega_{2}$ изотропньй конус $X^{1} X_{1}+X^{2} X_{2}=0$ несет два однопараметрических семейства двумерных плоских образующих. Эти семейства задаются соответственно системами

$$
\begin{array}{ll}
\lambda X^{1}=\mu X_{2}, & \lambda X^{2}=-\mu X_{1}, \\
\lambda X^{1}=\mu X^{2}, & \lambda X_{2}=-\mu X_{1} .
\end{array}
$$

Двумерное подмногообразие $\mathscr{L}$ является вполне изотропным, если все его касательные пространства являются плоскими образующими изотропных конусов, т. е. принадлежат либо семейству (90), либо семейству (91). В работе [12] (см. также [11], [14]) доказано, что $C O(2,2)$-структура на $\mathfrak{N}$ будет конформно-плоской, если (и только если) через каждую точку многообразия $M$ в любом вполне изотропном направлении проходит вполне изотропное подмногообразие. Эта структура будет конформно-полуплоской, если (и только если) выполняется только одно из следующих двух условий:

1) через каждую точку многообразия $M$ в любом вполне изотропном направлении семейства (90) проходит вполне изотропное подмногообразие;

2) через каждую точку многообразия $M$ в любом вполне изотропном направлении семейства (91) проходит вполне изотропное подмногообразие.

В работе [12] доказано, что четырехмерное многообразие $\mathfrak{N}$ невырожденных нуль-пар обладает свойствами:

1) через каждую точку многообразия $\mathfrak{N}$ в любом вполне изотропном направлении семейства (91) проходит вполне изотропное подмногообразие;

2 ) в каждой точке многообразия $\mathfrak{N}$ существует ровно два вполне изотропных направления, принадлежащих семейству (90), в направлении которых проходят вполне изотропные подмногообразия (эти подмногообразия являются плоскими образуюшими многообразия $\mathfrak{N})$.

Это означает, что $C O(2,2)$-структура на 4-мерном многообразии невырожденных нуль-пар является конформно-полуплоской.

Используя результаты предыдущего параграфа, можно сформулировать следующее утверждение, которое дает подробную характеристику вполне изотропных подмногообразий, сушествование которых доказано в [12]. 
СлЕДСТВИЕ 5. Пусть $\mathscr{L}$ - 2-мерное вполне изотропное подмногообразие 4-мерного многообразия невырожденных нуль-пар $\mathfrak{W}$. Тогда $\mathscr{L}$ - вполне геодезическое лагранжево подмногообразие. Подмногообразие $\mathscr{L}$ является либо плоской образующей, диффеоморфной $A_{2}$, либо аффинной областью на 2-мерном многообразии Сегре, диффеоморфной $A_{1} \times A_{1}^{*} \equiv A_{2}$, m. е. двумерной линейчатой квадрикой. Конструкции таких подмногообразий описаны $в$ примерах 1-3 при $n=2$. Через каждую точку многообразия $\mathfrak{W}$ проходят две плоские образующие, а множество вполне изотропных линейчатых квадрик, проходящих через эту точку, находится в биективном соответствии с әлементами множества одномерных подпространств двумерного векторного пространства (т.е. с точками проективной прямой).

\section{Список литературы}

1. Кириченко В. Ф. Обобщенная эрмитова геометрия в касательном расслоении // Изв. АН ЭстССР. Физика, математика. 1984. Т. 33. С. 363-368.

2. Кириченко В. $\Phi$. Касательное расслоение с точки зрения обобщенной эрмитовой геометрии // Изв. вузов. Математика. 1984. Т. 6. С. 50-58.

3. Кобаяши Ш., Номидзу К. Основы дифференциальной геометрии. М.: Наука, 1981.

4. Кириченко B. $\Phi$. Методы обобщенной эрмитовой геометрии в теории почти контактных многообразий // Итоги науки и техн. Проблемы геометрии. Т. 18. М.: ВИНИТИ АН CCCP, 1986. C. 25-71.

5. Kirichenko V. F. Generalized quasi-Kaehlerian manifolds and axioms of $C R$-submanifolds in generalized Hermitian geometry. II // Geometriae Dedicata. 1994. V. 52. P. 53-85.

6. Dube K. K. On almost hyperbolic Hermitian manifolds // Ann. Univ. Timishoara. Ser. Stinite Mat. 1973. V. 11. P. 47-54.

7. Libermann P. Sur le problèmé d'equivalence di certaines structure infinitesimales // Ann. di Matematica. 1951. V. 36. P. 247-261.

8. Gray A., Hervella L. M. The sixteen classes of almost Hermitian manifolds and their linear invariants // Ann. Math. Pure ed Appl. 1980. V. 123. № 4. P. 35-58.

9. Blair D. Contact manifolds in geometry // Lecture Notes in Math. 1976. V. 509. P. 1-145.

10. Kobayashi $S$. Principal fibre bundle with the 1-dimensional toroidal group // Tôhoku Math. J. 1956. V. 8. P. 29-45.

11. Akivis M. A, Goldberg V.V. Conformal differential geometry and its generalizations. N. Y.-Chichester-Brisbane-Toronto-Singapure: John Wiley \& Sons Inc., 1996.

12. Акивис М. А. О вполне изотропных подмногообразиях четырехмерной псевдоконформной структуры // Изв. вузов. Математика. 1983. № 1. С. 3-10.

13. Коннов В. В. Дифференциальная геометрия некоторых классов алгебраических многообразий. Самара: СамГПУ, 1998.

14. Акивис М. А., Коннов В. В. Некоторые локальные аспекты теории конформных структур // УМH. 1993. Т. 48. №1(289). С. 3-40. 